\title{
1 Topology-Specific Injectable Sticky Hydrogels
}

\author{
2 Mehdi Vahdati, Guylaine Ducouret, Costantino Cretor., and Dominique Hourdet*
}
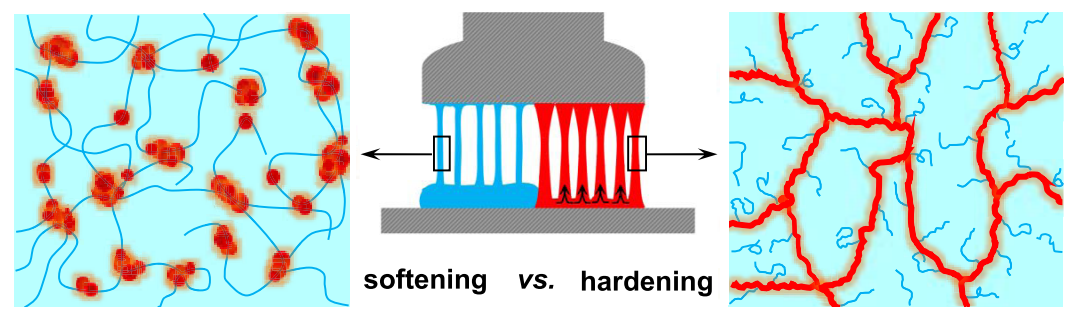

3 ABSTRACT: Stimuli-responsive injectable hydrogels based on weak supramolecular interactions may represent safer alternatives to 4 chemically reactive adhesive hydrogels for biomedical applications where weak to moderate adhesion is required. We investigated the 5 linear and nonlinear rheological properties as well as the adhesive properties of two thermoresponsive graft copolymers with inverse 6 topologies, poly $(N$-isopropyl acrylamide $)-g$-poly $(N, N$-dimethylacrylamide) (PNIPAM- $g$-PDMA) and PDMA- $g$-PNIPAM. Except for 7 their topologies, these copolymers are analogous in terms of chemistry, architecture (graft), and monomer composition (50-50 wt $8 \%)$. Over a wide range of concentrations, they both form injectable homogeneous solutions at room temperature and turn into soft 9 and sticky viscoelastic hydrogels close to body temperature. We find that the linear viscoelastic properties of these two hydrogels are 10 not discernible far above the thermal transition temperature. However, the PNIPAM-g-PDMA hydrogel having long 11 thermoresponsive backbones shows a strain-hardening behavior in large strains both in probe tack tests and in shear. The inverse 12 topology, PDMA-g-PNIPAM, showed no hardening and simply softened until failure. This distinction was observed regardless of the 13 polymer concentration (in the entangled regime). We attribute the hardening to a continuous, load-bearing nanostructure from 14 strong hydrophobic PNIPAM associations, while the softening is due to the easy pullout of short PNIPAM grafts from separate 15 hydrophobic clusters bridged by PDMA backbones. The findings of this work highlight the importance of macromolecular design in 16 determining the nanostructure and thereby the mechanical performance of soft hydrogels for specific applications.

\section{INTRODUCTION}

17 Hydrogels are generically similar to many living tissues in 18 terms of water content (up to $98 \mathrm{wt} \%$ ) and softness (typical 19 moduli in the range of $\left.10^{1}-10^{4} \mathrm{~Pa}\right)$ and are therefore useful for 20 a host of biomedical and pharmaceutical applications. ${ }^{1-3}$ In 21 this context, injectable solutions that turn into hydrogels inside 22 the body are useful for specific applications such as targeted 23 delivery of bioactive agents and living cells, bioprinting, and 24 minimally invasive tissue approximation, among others. ${ }^{1,4-6}$

25 One possibility is in situ chemical (covalent) cross-linking of 26 injectable formulations based on monomer or polymer 27 solutions containing polymerizable functional groups. ${ }^{7-10}$ 28 Although widely used, this strategy can be limited in its 29 scope due to concerns for the toxicity of the chemicals used 30 (monomers, initiators, etc.) and/or the chemical reactions 31 involved (free radicals, reaction heat, etc.) inside the body. ${ }^{7,9}$ 32 An appealing alternative is using weaker supramolecular 33 interactions that can be switched on in response to an external 34 stimulus such as body temperature, salt concentration, or $\mathrm{pH}$ 35 to form physical hydrogels in situ. ${ }^{11-13}$ Such stimuli-responsive 36 hydrogels usually offer reversible gelation and allow on37 demand switchability and removability when such features are 38 desired. $^{12-14}$
Depending on their function, hydrogels will see different 39 kinds of mechanical stress during their service life and may 40 very well experience deformations outside the small strain 41 (linear) regime. ${ }^{3,15}$ It is thus important, for optimal design, to 42 understand the nonlinear behavior of these soft materials in 43 large strains. ${ }^{16-18}$ Nonlinear mechanical properties of chemical 44 hydrogels can be measured using conventional experiments 45 such as uniaxial tension or compression tests. Moreover, by 46 drawing analogies with hydrophobic systems like cross-linked 47 rubbers and using the theory of rubber elasticity, the nonlinear 48 behavior of these materials can be linked to their (nano)- 49 structure and/or linear mechanical properties, and vice versa. ${ }^{3} 50$ However, the nonlinear behavior of physically cross-linked 51 hydrogels is far less studied and understood, possibly due to 52 the difficulty of performing conventional nonlinear character- 53 ization experiments on these materials. For instance, it is 54 
55 challenging to perform elongational rheology or uniaxial 56 tension experiments on thermoresponsive hydrogels that are 57 in the sol (liquid) state at room temperature.

58 Nonetheless, temperature remains a popular stimulus due to 59 its practical convenience. Polymers featuring the so-called 60 lower critical solution temperature (LCST) in water close to 61 body temperature are particularly interesting for biomedical 62 applications. ${ }^{19,20}$ Poly $(N$-isopropyl acrylamide) (PNIPAM) is 63 the archetype of an LCST polymer featuring a sharp coil-to64 globule transition upon heating above $32{ }^{\circ} \mathrm{C} .{ }^{12,19,21,22}$ The 65 LCST of PNIPAM is almost independent of the polymer 66 concentration and molecular weight and usually leads to 67 abrupt, macroscopic phase separation, also called synere68 sis. $^{22-25}$ For this reason, PNIPAM can be copolymerized with 69 a hydrophilic comonomer to produce stable thermoswitchable 70 associative polymers (physical hydrogels) with no volume 71 transition. ${ }^{26-28}$

72 Guo et al. investigated relationships between nanostructures 73 and mechanical properties of chemically and physically cross74 linked hydrogels based on graft copolymers of PNIPAM and 75 hydrophilic poly $\left(N, N\right.$-dimethylacrylamide) (PDMA). ${ }^{29,30}$ To 76 specify the role of topology, they studied PDMA-g-PNIPAM 77 and PNIPAM-g-PDMA with inverse topologies by keeping the 78 chemistry, architecture (grafted), and monomer composition 79 (near 50-50) the same.

80 Despite some differences in their thermal phase transition, 81 these chemically cross-linked hydrogels showed similar 82 enhancements of their modulus when heated above the 83 LCST of PNIPAM. ${ }^{29,31}$ In other words, they were not 84 distinguishable based on their linear dynamic behavior far 85 above the transition temperature. However, when studied in 86 large strain, the degree of thermal toughening was significantly 87 larger in the case of the hydrogel with PNIPAM as the cross88 linked network compared to its inverse topology. This 89 difference was mainly due to extensive bifurcation of the 90 crack in cross-linked PNIPAM-g-PDMA during fracture 91 experiments. $^{27,31}$ With insights from small-angle neutron 92 scattering (SANS), the nontrivial difference in large-strain 93 mechanical properties between the two hydrogels was ascribed 94 to the formation of a continuous nanostructure among 95 hydrophobic PNIPAM associations, while the same content 96 of PNIPAM as grafts led to separate aggregates. This scenario 97 was backed by the affine deformation observed for the 98 PNIPAM-rich domains under uniaxial deformation. ${ }^{29,31}$

99 The analogous solutions showed some differences in their 100 thermal phase transition, with a more abrupt transition having 101 a higher enthalpy in the case of PNIPAM-g-PDMA. ${ }^{30}$ 102 However, and similar to their chemically cross-linked counter103 parts, the two solutions eventually had comparable linear gel104 like viscoelastic properties when heated above the LCST of 105 PNIPAM. In the case of PNIPAM backbones, the authors 106 suggested a continuous nanostructure from hydrophobic 107 associations to account for the formation of a stable gel. 108 However, without nonlinear mechanical experiments, it was 109 not possible to distinguish between separate hydrophobic 110 clusters or a continuous nanostructure in the case of the 111 inverse topology, PDMA-g-PNIPAM.

112 Our most recent work marks the first instance of nonlinear 113 mechanical experiments on such physical hydrogels where we 114 investigated a large-molecular-weight PNIPAM- $g$-PDMA re115 sponsive copolymer as a soft adhesive. ${ }^{32}$ The polymer solution 116 was injectable below body temperature and turned into a 117 viscoelastic sticky hydrogel above the LCST of PNIPAM. The performance of this hydrogel was remarkable in both air and 118 water, mainly due to the formation of stable fibrils due to strain 119 hardening at large deformations in a temperature-controlled 120 probe tack experiment. This nontrivial strain hardening was 121 attributed to a load-bearing hydrophobic nanoscaffold across 122 the soft, swollen PDMA matrix. However, the inverse topology 123 has never been studied in large strains. In addition, the 124 universality of this behavior in other testing geometries and at 125 other copolymer concentrations is unknown.

Therefore, the aim of the present work is to establish a link 127 between the linear and nonlinear mechanical properties and 128 the nanostructure of physical hydrogels of PDMA-g-PNIPAM 129 and PNIPAM-g-PDMA, both at 50-50 weight composition, 130 based on inverse topologies. For this, their thermal association 131 and dynamic behaviors are first studied to obtain as much 132 information about their internal structures as possible. We will 133 then focus on potential differences in their nonlinear 134 mechanical behavior, i.e., when they are in large strains. The 135 first experiment used is a probe tack procedure specifically 136 adapted to a rheometer for these materials. This test studies 137 the nonlinear properties of a thin, highly confined layer of an 138 adhesive (the hydrogels in this case) under the tensile loading 139 mode. The effect of different experimental parameters 140 including the polymer concentration and level of confinement 141 (thickness) will be studied for both topologies. To investigate 142 the validity of our findings regardless of the testing geometry 143 used, the hydrogels will be studied in the so-called stress 144 growth experiments, where they are sheared to very large 145 strains at different constant shear rates.

146

\section{EXPERIMENTAL SECTION}

2.1. Materials. All of the materials were used as received. $N, N-147$ Dimethylacrylamide (DMA), $N$-isopropylacrylamide (NIPAM), 148 acrylic acid (AA), 2-aminoethanethiol hydrochloride (AET-HCl), 149 dicyclohexylcarbodiimide (DCCI), potassium persulfate (KPS), 150 $N, N, N^{\prime}, N^{\prime}$-tetramethylethylenediamine (TEMED), and 1-methyl-2- 151 pyrrolidone (NMP) were purchased from Sigma-Aldrich and Merck. 152

2.2. Synthesis. The synthesis of the graft copolymers was adapted 153 from previous work. ${ }^{30}$ Following a grafting through procedure, the 154 side chains were first synthesized in the form of amino-terminated 155 telomers by free radical polymerization. The amino end group was 156 then turned into a double bond via a coupling reaction with the 157 carboxylic group of acrylic acid in an organic solvent (NMP). The 158 purified and freeze-dried macromonomers (chains with a terminal 159 double bond) were then copolymerized in a 50-50 weight ratio with 160 the other monomers in water to give graft copolymers. For instance, 161 starting with NIPAM, we first prepared PNIPAM macromonomers, 162 which were then used to produce PDMA grafted with PNIPAM, or 163 PDMA-g-PNIPAM. A more detailed explanation of the reaction 164 conditions and formulations is given in the Supporting Information 165 (SI).

2.3. Preparation of Solutions. Aqueous solutions of the purified 167 copolymers were prepared at 4, 8, and 16 wt \% (by total weight of the 168 polymer) by stirring at room temperature. They were then left in a 169 fridge for $24 \mathrm{~h}$ to obtain bubble-free solutions. This was particularly 170 necessary in the case of $16 \mathrm{wt} \%$ PNIPAM-g-PDMA due to its high 171 viscosity. In all of the cases, the solutions were eventually 172 homogeneous and transparent at room temperature.

173

2.4. ${ }^{1} \mathrm{H}$ NMR Analysis. The macromonomers and the graft 174 copolymers were studied using ${ }^{1} \mathrm{H}$ NMR in deuterated water $\left(\mathrm{D}_{2} \mathrm{O}, 175\right.$ solvent peak at $\delta \approx 4.79 \mathrm{ppm}$ ) on a Bruker Avance III $400 \mathrm{MHz} 176$ NMR spectrometer. In the case of the macromonomers, the 177 experiment was carried out to ensure the presence of terminal double 178 bonds. The three hydrogens of the terminal double bonds appear 179 between 5.5 and $6.5 \mathrm{ppm}$. In the case of PNIPAM, the single $\mathrm{H}$ on the 180 tertiary carbon and the six hydrogens of the two methyl groups appear 181 
182 at 3.9 and $1.13 \mathrm{ppm}$, respectively, while in the case of PDMA, the 183 dimethyl hydrogens appear in the vicinity of $2.9-3.1 \mathrm{ppm}$. In both 184 cases, the backbone hydrogens appear between 1.4 and $2.7 \mathrm{ppm}$ in 185 two sets of peaks. The same chemical shifts were used to determine 186 the molar ratio between NIPAM and DMA units in the copolymers. 187 This was then turned into weight ratios for each copolymer. The ${ }^{1} \mathrm{H}$ 188 NMR spectra of one of the macromonomers and one of the 189 copolymers are presented in Figures S1 and S2 as examples.

190 2.5. Size Exclusion Chromatography (SEC). SEC measure191 ments on the side chains were performed at $28^{\circ} \mathrm{C}$ in tetrahydrofuran 192 (THF) containing $2 \mathrm{wt} \%$ trimethylamine as the mobile phase (at 0.6 $193 \mathrm{~mL} \cdot \mathrm{min}^{-1}$ ) on a VISCOTEK GPCmax (VE $2001 \mathrm{GPC}$ ) equipped 194 with a Viscotek triple detector (TDA 302). The characterization of 195 graft copolymers was performed in dimethylformamide (DMF) at 35 $196{ }^{\circ} \mathrm{C}$ using an EcoSEC (TOSOH Bioscience) equipped with a refractive 197 index (RI) detection system. The flow rate was controlled at $0.5 \mathrm{~mL}$. $198 \mathrm{~min}^{-1}$ using DMF with $\mathrm{LiCl}(5 \mathrm{mM})$ as the mobile phase. The 199 concentration of the injected samples was approximately $5 \mathrm{mg} \cdot \mathrm{mL}^{-1}$, 200 and the samples were not filtered prior to injection. The column had a 201 cutoff at around $3 \mathrm{MDa}$ according to the manufacturer.

202 2.6. Differential Scanning Calorimetry (DSC). The thermody203 namic phase transition of PNIPAM in water was determined using a 204 DSC Q200 (TA Instruments). For all of the samples studied, around $20540 \mathrm{mg}$ of polymer solution at $8 \mathrm{wt} \%$ (total (co)polymer) was placed 206 in a measurement pan, while the reference pan was filled with the 207 corresponding amount of water in the sample. After $10 \mathrm{~min}$ of 208 equilibration at $20^{\circ} \mathrm{C}$, the samples were heated, cooled, and heated 209 again at a rate of $2{ }^{\circ} \mathrm{C} \cdot \mathrm{min}^{-1}$ between 20 and $60{ }^{\circ} \mathrm{C}$ to remove any 210 possible thermal history. The DSC thermograms presented in this 211 work are from the last heating ramp. It has been previously validated 212 that this heating rate is slow enough to ensure proximity to 213 thermodynamic equilibrium at all of the studied temperatures. ${ }^{30}$

214 2.7. Small-Angle Neutron Scattering (SANS). SANS experi215 ments were performed at Laboratoire Léon Brillouin (CEA Saclay, 216 France) on a PACE spectrometer. The wavelength of the incident 217 neutron beam was set at $\lambda=5.0 \AA$ with a corresponding sample-to218 detector distance of $5.0 \mathrm{~m}$. This configuration provides a scattering 219 vector modulus $[q=(4 \pi / \lambda) \sin (\theta / 2)]$ ranging between 0.008 and $2200.09 \AA^{-1}$ (where $\theta$ is the scattering angle). All of the samples were 221 prepared at room temperature in $\mathrm{D}_{2} \mathrm{O}$ and transferred to $2 \mathrm{~mm}$ thick 222 quartz containers for SANS experiments. For the data treatment, the 223 scattering from the empty quartz cell was subtracted, the efficiency of 224 the detector cell was normalized by the intensity delivered by a pure 225 water cell of $1 \mathrm{~mm}$ thickness, and absolute measurements of the 226 scattering intensity $I(q)\left(\mathrm{cm}^{-1}\right)$ were obtained from the direct 227 determination of the incident neutron flux and the cell solid angle.

228 2.8. Linear Rheology. Linear rheology measurements were 229 performed on Thermo Scientific HAAKE RheoStress 600 (Thermo 230 Fisher Scientific), which is a stress-controlled rheometer. A cone-plate 231 geometry was used in all of the measurements, where the cone angle 232 and diameter were $2^{\circ}$ and $20 \mathrm{~mm}$, respectively. The required amount 233 of the solution to be tested was placed on the bottom plate at $20^{\circ} \mathrm{C}$ 234 followed by contact with the cone. The entire geometry was sealed 235 using a custom-built vapor trap to minimize evaporation. Preliminary 236 amplitude sweeps confirmed that all of the samples were in their linear 237 viscoelastic regime at a stress of $2 \mathrm{~Pa}$ (see Figure S3). Temperature 238 sweeps were then performed by heating the samples up to $60^{\circ} \mathrm{C}$ at a 239 rate of $2{ }^{\circ} \mathrm{C} \cdot \mathrm{min}^{-1}$ at $2 \mathrm{~Pa}$ and $1 \mathrm{~Hz}$. As the cooling runs, performed at 240 the same rate to the initial temperature, show the same viscoelastic 241 profile with negligible hysteresis, we will only use the heating run in 242 the following. The samples at 8 wt \% were also tested in frequency 243 sweeps at low $\left(20^{\circ} \mathrm{C}\right)$ and high $\left(50{ }^{\circ} \mathrm{C}\right)$ temperatures.

244 2.9. Probe Tack Test. The tackiness of soft adhesives such as 245 pressure-sensitive adhesives (PSAs) is typically studied on a probe 246 tack testing machine. ${ }^{15,33}$ However, the standard procedure is not well 247 suited to thermoresponsive hydrogels with large water contents like 248 those in this work. We adapted a DHR-3 (TA Instruments) 249 rheometer equipped with a sufficiently sensitive axial load cell (0.1 $250 \mathrm{~N}$ ) and a precise Peltier plate temperature control system to perform 251 probe tack experiments. A step-by-step scheme of the procedure is shown in Figure 1. We note that the very soft nature of the adhesives $252 \mathrm{fl}$ and the confinement $\left(r \gg h_{0}\right.$, with $r$ and $h_{0}$ being the radius of the 253

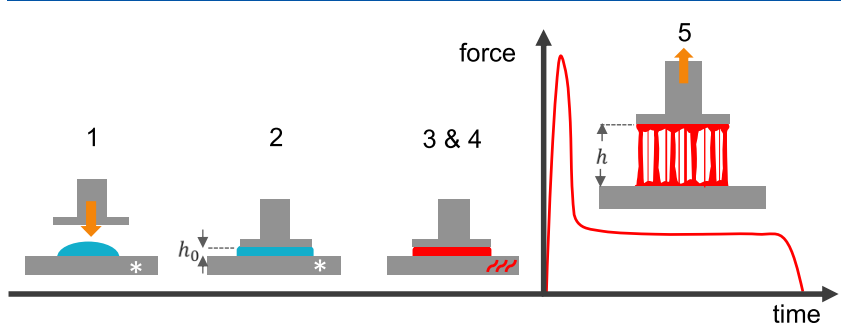

Figure 1. Schematic representation of the steps involved in the new probe tack procedure adapted to a rheometer.

probe and the thickness of the layer, respectively) distinguish this test 254 from a uniaxial tensile test, which measures the strength of long, 255 dumbbell-shaped samples of more solidlike materials with small 256 widths $(w)$ and thicknesses $(t)$ compared to their lengths $(l>w$ and 257 t). ${ }^{34}$

A flat, sand-blasted probe attached to the axial load cell of the 259 rheometer and a sand-blasted plate mounted on the Peltier 260 temperature control system were used as the top and bottom plates, 261 respectively. Both plates were made of stainless steel as a simple 262 model surface. The bottom plate was always maintained at $20{ }^{\circ} \mathrm{C} 263$ during steps 1 and 2 in Figure 1. The required volume of the solution, 264 estimated from the initial thickness of the film $\left(h_{0}=200-800 \mu \mathrm{m}\right) 265$ and the radius of the probe $(r=10 \mathrm{~mm})$, was placed on the bottom 266 plate using a micropipette at $20^{\circ} \mathrm{C}$. Unlike a typical probe tack test on 267 more solidlike adhesive samples where the layer is slightly pressed by 268 the probe to ensure intimate contact, the injectable solutions were in 269 the liquid state and good contact was simply achieved by squeezing 270 the solution to the intended initial thickness (step 2).

The layer was then heated up quickly to the target temperature ( $\begin{array}{ll}50 & 272\end{array}$ ${ }^{\circ} \mathrm{C}$, unless otherwise mentioned) followed by a $10 \mathrm{~min}$ dwell time to 273 make sure the temperature is homogeneous across the hydrogel (steps 274 3 and 4, Figure 1). All of the experiments were started at zero residual 275 force. Eventually, the axial force required to detach the probe at a 276 constant debonding rate $\left(V_{\mathrm{deb}}\right)$ was recorded as a function of time (or 277 displacement; step 5). Nominal stress $(\sigma)$, defined as the measured 278 force $(F)$ divided by the initial contact area $\left(A_{0}\right)$, and nominal strain 279 $(\varepsilon)$, defined as the displacement normalized by the initial thickness, 280 were then calculated from the raw data. A nominal strain rate $(\dot{\varepsilon})$ can 281 be defined as the debonding rate normalized by the initial thickness. 282

Apart from the useful information obtained from the shape of the 283 nominal stress-strain curves, the work of adhesion $\left(W_{\text {adh }}\right)$ provides a 284 useful means of making universal comparisons between different 285 samples. This parameter is defined as the energy required to make the 286 unit surface area of the adhesive and is calculated from the product of 287 the initial thickness and the area under the nominal stress-strain 288 curve up to failure.

289

We carefully investigated the reproducibility of our probe tack 290 experiments performed on the rheometer, as detailed in the SI (see 291 Figures S4 and S5).

2.10. Stress Growth. The nonlinear stress growth experiments 293 were performed on a DHR-3 rheometer (TA Instruments). Sampling 294 was done at $20{ }^{\circ} \mathrm{C}$, but the experiments were all performed under a 295 vapor trap at $50{ }^{\circ} \mathrm{C}$ after $5 \mathrm{~min}$ to ensure complete gelation and 296 equilibrium. Similar to the linear rheology, a cone and plate 297 configuration $\left(2^{\circ}, 20 \mathrm{~mm}\right)$ was used in all of the experiments to 298 apply uniform, constant shear rates of $0.025,0.1$, and $0.25 \mathrm{~s}^{-1}$ to the 299 entire sample beyond failure. In this geometry, strain is defined as the 300 product of strain rate and time (in $\mathrm{rad} \cdot \mathrm{rad}^{-1}$ ) equal to the 301 displacement of the cone divided by the cone angle (both in rad). 302 As such, a strain of $1000 \%$ corresponds to a displacement of $20^{\circ}$ (or 303 $0.35 \mathrm{rad}$ for a cone angle of $0.035 \mathrm{rad}$ ). 
Table 1. Summary of Synthesis Results for the Macromonomers (Grafts) and the Copolymers

\begin{tabular}{|c|c|c|c|c|c|}
\hline \multirow[b]{2}{*}{ copolymer } & \multicolumn{2}{|c|}{$M_{\mathrm{n}}\left(\mathrm{kg} \cdot \mathrm{mol}^{-1}\right)(\mathrm{PDI})$} & \multirow[b]{2}{*}{ NIPAM/DMA (wt \% feed) } & $\S$ & \multirow[b]{2}{*}{ grafts per chain } \\
\hline & graft $^{a}$ & copolymer $^{b}$ & & NIPAM/DMA (wt \% copolymer) $)^{c}$ & \\
\hline PDMA-g-PNIPAM & $8(1.4)$ & $320(4.2)$ & $50: 50$ & $50: 50$ & $\sim 20$ \\
\hline PNIPAM-g-PDMA & $14(1.2)$ & $880(2.0)$ & $50: 50$ & $50: 50$ & $\sim 30$ \\
\hline
\end{tabular}

${ }^{a} \mathrm{SEC}$ in THF. ${ }^{b} \mathrm{SEC}$ in DMF. ${ }^{c 1} \mathrm{H}$ NMR in $\mathrm{D}_{2} \mathrm{O}$.

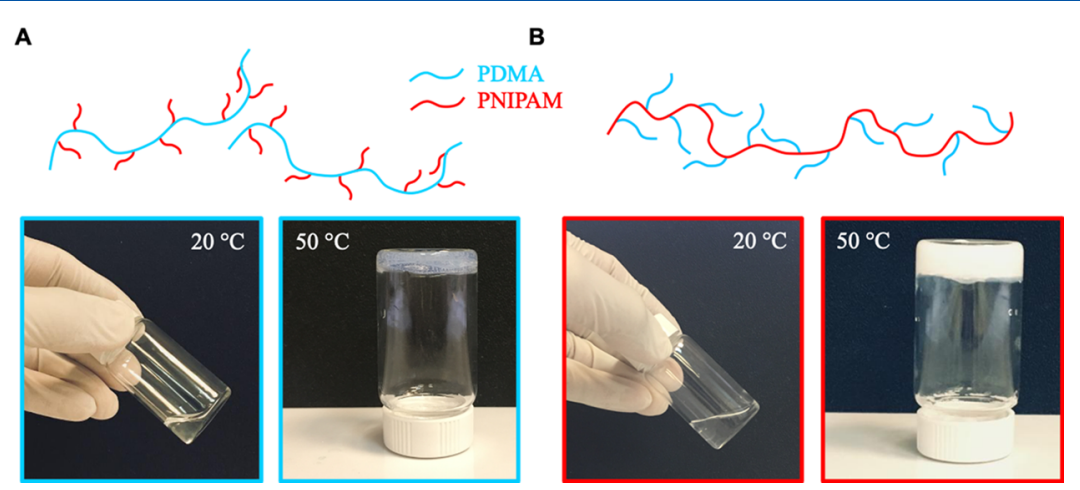

Figure 2. Schematic representation of the molecular architecture of (A) PDMA- $g$-PNIPAM and (B) PNIPAM- $g$-PDMA. The pictures below are from their $8 \mathrm{wt} \%$ solutions and hydrogels at 20 and $50{ }^{\circ} \mathrm{C}$, respectively. The color code used in the following is based on the backbone, i.e., blue for PDMA- $g$-PNIPAM and red for PNIPAM- $g$-PDMA.

\section{RESULTS AND DISCUSSION}

305 3.1. Synthesis of Inverse Topologies. To pin down the 306 impact of the macromolecular morphology on the nonlinear 307 behavior of physical hydrogels, we designed two copolymers 308 with inverse topologies, PNIPAM-g-PDMA and PDMA-g309 PNIPAM, by keeping the same chemistry, the same grafted 310 architecture, and the same weight composition. We have 311 reported on the linear viscoelastic properties of such hydrogels 312 in previous work. ${ }^{30}$ As shown in Table 1 , the two copolymers 313 were found, within the sensitivity limits of ${ }^{1} \mathrm{H}$ NMR, to have 314 effectively the same weight composition in line with the weight 315 feed ratio (50:50). See Figure S2 for the NMR spectrum as an 316 example.

317 Concerning the macromolecular structure, some differences 318 can be highlighted, mainly related to the size of individual 319 components. The main difference originates from the molar 320 mass of the backbones: $M_{\mathrm{n}}=320 \mathrm{~kg} \cdot \mathrm{mol}^{-1}$ for PDMA-g321 PNIPAM and $880 \mathrm{~kg} \cdot \mathrm{mol}^{-1}$ for PNIPAM-g-PDMA. As the size 322 of the grafts is also different for PNIPAM $\left(M_{\mathrm{n}}=8 \mathrm{~kg} \cdot \mathrm{mol}^{-1}\right)$ 323 and PDMA $\left(M_{\mathrm{n}}=14 \mathrm{~kg} \cdot \mathrm{mol}^{-1}\right)$, the average number of side 324 chains per backbone is 20 for PDMA-g-PNIPAM and 30 for 325 PNIPAM-g-PDMA. We also noted a small fraction (around 5 $326 \mathrm{wt} \%$ ) of unreacted PDMA grafts in the SEC traces of 327 PNIPAM-g-PDMA probably due to the free radical copoly328 merization of the PDMA macromonomer with the NIPAM 329 monomer. However, this shall not impact the thermores330 ponsive behavior of PNIPAM backbones, as will be discussed 331 in Section 3.2.1. Schematic pictures of the structures of the 332 copolymers as well as images of their formulations at $8 \mathrm{wt} \%$ in 333 the liquid and gel states are presented in Figure 2.

334 Despite these structural differences, the main parameter in 335 the foreground, especially at high temperatures, is the 336 PNIPAM composition that is comparable for the two 337 copolymers. While the phase transition of homopolymer 338 PNIPAM is only marginally dependent on its molecular 339 weight, ${ }^{35,36}$ the main idea behind this work is to compare the 340 linear and nonlinear viscoelastic properties triggered by the 341 association of PNIPAM moieties, either backbones of several hundreds of $\mathrm{kg} \cdot \mathrm{mol}^{-1}$ or side chains of several $\mathrm{kg} \cdot \mathrm{mol}^{-1}$. In 342 other words, the main role of the PDMA chains (in blue in 343 Figure 2) is to avoid the macroscopic phase separation of the 344 copolymer (called syneresis) at high temperatures. This 345 explains why we chose to work at the fixed monomer 346 composition of 50:50.

By comparison with our previous work, ${ }^{30}$ we have used a 348 lower ratio of the initiator to the monomer in the free radical 349 copolymerization step (see Synthesis of the Graft Copolymers 350 in the SI) to get copolymers with larger molecular weights. 351 This actually serves the purpose of this work that focuses on 352 nonlinear mechanical properties. Higher molecular weights and 353 larger numbers of grafts per chain favor interchain interactions 354 and entanglements and entail lower overlap concentrations for 355 the formation of a percolated network. ${ }^{30}$

3.2. Thermoresponsive Assemblies. As reported pre- 357 viously, thermoresponsive graft copolymers designed with an 358 equilibrated composition between hydrophilic and PNIPAM 359 sequences do not macroscopically phase separate in semidilute 360 solutions. ${ }^{30,32,37}$ Indeed, this is the case with our copolymers 361 tailored with $50 \mathrm{wt} \%$ each sequence, which allows us to 362 investigate their properties over a wide range of concentrations 363 and temperatures. Given that the hydrogels obtained from 364 these copolymer solutions are merely physically cross-linked 365 above the transition temperature, it is reasonable to expect that 366 their mechanical properties strongly depend on the phase 367 separation dynamics and the final morphology of the 368 associations.

3.2.1. Thermal Phase Transition. The disruption of the 370 hydrogen bonds between water molecules and amide groups of 371 NIPAM units as well as their reorganization are endothermic 372 processes together marking the coil-to-globule transition of 373 PNIPAM. The enthalpy of this transition, which accounts for 374 the reorganization of hydrogen bonds, can vary quite widely 375 depending on the initial polymer concentration but typical 376 values between 4 and $6 \mathrm{~kJ} \cdot \mathrm{mol}^{-1}$ of NIPAM are often reported 377 for dilute and semidilute solutions of linear chains $(C<10 \mathrm{wt} 378$ $\%){ }^{24,36,38,39}$ As documented in the literature, this is a sharp 379 
380 transition starting at around $32-34{ }^{\circ} \mathrm{C}$ for the PNIPAM 381 homopolymer. Unlike many other LCST polymers (e.g., 382 poly(vinyl caprolactam), PVCL, or poly(ethylene oxide) 383 (PEO)) having a classical Flory-Huggins temperature384 concentration phase diagram, the LCST of linear PNIPAM is 385 weakly dependent on the molecular weight and polymer 386 concentration. $^{22,35,40-42}$

387 Figure 3 compares the thermal behavior of the two 388 copolymers at $8 \mathrm{wt} \%$ total polymer concentration (4 wt \%

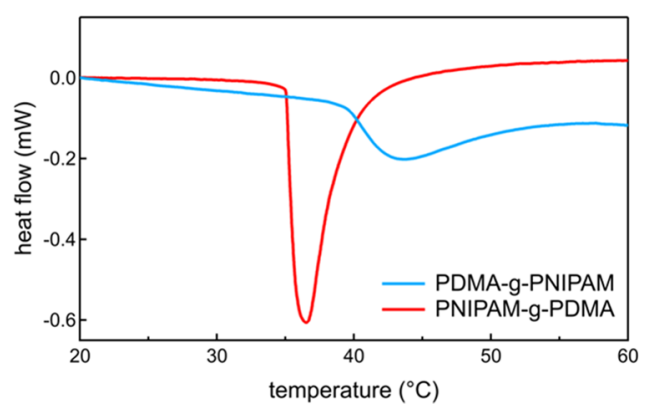

Figure 3. DSC thermograms showing heat flow as a function of temperature increase at $2{ }^{\circ} \mathrm{C} \cdot \mathrm{min}^{-1}$ for $8 \mathrm{wt} \%$ PDMA-g-PNIPAM and PNIPAM- $g$-PDMA in water.

389 PNIPAM) upon heating at $2{ }^{\circ} \mathrm{C} \cdot \mathrm{min}^{-1}$. Given their identical 390 nature, differences in the thermal behavior of the two 391 topologies are striking. With the copolymer bearing the 392 PNIPAM side chains, PDMA- $g$-PNIPAM, the transition takes 393 place at a relatively higher temperature (39.6 versus $35^{\circ} \mathrm{C}$ ), 394 with an enthalpy $60 \%$ lower compared to that of the inverse 395 topology, PNIPAM- $g$-PDMA. The transition is also broader, 396 taking place above $14^{\circ} \mathrm{C}$, while that of PNIPAM- $g$-PDMA is 397 quite sharp (above $8{ }^{\circ} \mathrm{C}$ ).

398 We note that the PDMA-g-PNIPAM thermogram in Figure 3993 clearly shows the absence of unreacted PNIPAM grafts. If 400 this were the case, free PNIPAM macromonomers would 401 phase separate at a lower temperature, leading to a first distinct 402 peak (around $35^{\circ} \mathrm{C}$ ) before that of the grafted PNIPAM 403 (around $40{ }^{\circ} \mathrm{C}$ ). As for the inverse topology, we cannot use 404 this argument because the side chains are not thermores405 ponsive. Yet, ungrafted (free) hydrophilic chains do not impact 406 the thermal association behavior of the PNIPAM backbone as 407 long as a 50:50 weight composition is maintained. ${ }^{31}$

408 It is insightful to draw a comparison between these 409 copolymers and the PNIPAM macromonomer precursor, as 410 well as a longer PNIPAM homopolymer $\left(M_{\mathrm{n}}=130 \mathrm{~kg} \cdot \mathrm{mol}^{-1}\right.$, 411 PDI = 1.5). The corresponding DSC thermograms can be 412 found in Figure S6 in the SI. Table 2 summarizes all of the 413 DSC data from our experiments. The first point to highlight is 414 that both graft copolymer solutions show delayed onsets of the 415 thermal transition by almost $4{ }^{\circ} \mathrm{C}$ compared to their ungrafted 416 counterparts. The delay in the thermal transition is attributed 417 to the steric hindrance and higher loss of entropy of the 418 hydrophilic PDMA sequences either present within the 419 backbone or as pendant chains. The impact of the architecture 420 on the phase separation process has been widely studied. For 421 instance, higher cloud points and lower transition enthalpies 422 have been reported for cyclic and star homopolymers of 423 PNIPAM compared to linear chains of similar molar masses. In 424 the case of copolymers, the architecture and the nature of the 425 comonomer and its distribution are critical with a major effect 426 when hydrophilic units are randomly distributed along a
Table 2. Summary of DSC Experiments Performed on PDMA-g-PNIPAM and PNIPAM-g-PDMA Copolymers (from Figure 3) and Compared with the PNIPAM Macromonomer and a PNIPAM Homopolymer (from Figure $S 6$ in the $S I)^{a}$

\begin{tabular}{|c|c|c|c|c|c|}
\hline \multicolumn{3}{|c|}{ PDMA-g-PNIPAM } & \multicolumn{3}{|c|}{ PNIPAM-g-PDMA } \\
\hline $\begin{array}{l}T_{\text {onset }} \\
\left({ }^{\circ} \mathrm{C}\right)\end{array}$ & $\begin{array}{l}T_{\text {dip }} \\
\left({ }^{\circ} \mathrm{C}\right)\end{array}$ & $\frac{\Delta H}{\left(\mathrm{~kJ} \cdot \mathrm{mol}_{\text {NIPAM }}{ }^{-1}\right)}$ & $\begin{array}{l}T_{\text {onset }} \\
\left({ }^{\circ} \mathrm{C}\right)\end{array}$ & $\begin{array}{l}T_{\text {dip }} \\
\left({ }^{\circ} \mathrm{C}\right)\end{array}$ & $\begin{array}{c}\Delta H \\
\left(\mathrm{~kJ} \cdot \mathrm{mol}_{\mathrm{NIPAM}}{ }^{-1}\right)\end{array}$ \\
\hline 39.6 & 43.5 & 1.8 & 35.0 & 36.5 & 4.5 \\
\hline \multicolumn{3}{|c|}{ PNIPAM macromonomer } & \multicolumn{3}{|c|}{ homopolymer PNIPAM } \\
\hline $\begin{array}{l}T_{\text {onset }} \\
\left({ }^{\circ} \mathrm{C}\right)\end{array}$ & $\begin{array}{c}T_{\text {dip }} \\
\left({ }^{\circ} \mathrm{C}\right)\end{array}$ & $\frac{\Delta H}{\left(\mathrm{~kJ} \cdot \mathrm{mol}_{\mathrm{NIPAM}}{ }^{-1}\right)}$ & $\begin{array}{l}T_{\text {onset }} \\
\left({ }^{\circ} \mathrm{C}\right)\end{array}$ & $\begin{array}{l}T_{\text {dip }} \\
\left({ }^{\circ} \mathrm{C}\right)\end{array}$ & $\begin{array}{c}\Delta H \\
\left(\mathrm{~kJ} \cdot \mathrm{mol}_{\text {NIPAM }}{ }^{-1}\right)\end{array}$ \\
\hline 35.0 & 37.1 & 4.5 & 30.9 & 32.4 & 4.4 \\
\hline
\end{tabular}

${ }^{a}$ Standard deviations in the reported temperatures and enthalpies did not exceed $0.1{ }^{\circ} \mathrm{C}$ and $7.5 \%$, respectively.

PNIPAM chain, as compared to block or graft architectures. In 427 graft copolymers, the hydrophilicity (or hydrophobicity) of the 428 comonomer plays a determining role in the phase behavior 429 with more hydrophilic comonomers such as charged 430 polyelectrolytes generally having a much stronger effect than 431 neutral comonomers on the association temperature. ${ }^{19,41,43} 432$

Another important observation from Table 2 is that the 433 transition enthalpy per NIPAM unit is almost the same $(4.5 \mathrm{~kJ} \cdot 434$ mol $_{\text {NIPAM }}{ }^{-1}$ ) for the two homopolymers as well as PNIPAM-g- 435 PDMA, in good agreement with data reported for linear 436 PNIPAM in this concentration range. However, it drops 437 significantly (by 60\%) for PDMA-g-PNIPAM. Considering 438 that the enthalpy can be correlated to the effective balance 439 between disruption (water-amide) and reformation (water- 440 water and amide-amide) of hydrogen bonds, the lower energy 441 observed for the aggregation of relatively short PNIPAM 442 chains $\left(8 \mathrm{~kg} \cdot \mathrm{mol}^{-1}\right)$ covalently bound to the PDMA backbone 443 can be related to (1) the energy barrier against the association 444 process due to the steric hindrance imposed by the water- 445 soluble backbone and/or (2) the formation of smaller 446 PNIPAM aggregates with a "hydrated" shell. 447

Similar observations have been reported by our group, either 448 with linear graft copolymers or cross-linked networks, where 449 placing the PNIPAM on the backbone facilitates phase 450 separation at lower temperatures. ${ }^{29-31}$ Overall, the DSC 451 analysis shows that, the chemistry, architecture, and 452 composition being the same, having a long PNIPAM 453 backbone, as opposed to short PNIPAM side chains, favors 454 the thermal phase separation as shown by a sharper transition 455 of a higher enthalpy occurring at a lower temperature. $\quad 456$

3.2.2. Phase Morphology above Transition. The morphol- 457 ogy of copolymer formulations induced by the thermal phase 458 separation of PNIPAM on a local scale was investigated by 459 small-angle neutron scattering using $\mathrm{D}_{2} \mathrm{O}$ as the solvent to 460 enhance the scattering contrast between the two phases. It has 461 been previously validated that the behavior of PNIPAM 462 remains much the same in $\mathrm{D}_{2} \mathrm{O}$ as in $\mathrm{H}_{2} \mathrm{O}$, with a $1-2{ }^{\circ} \mathrm{C}$ shift 463 in the transition temperature. ${ }^{30,44}$ Figure 4 shows the double- $464 \mathrm{f4}$ logarithmic plots of the measured scattering intensity, $I$, as a 465 function of the scattering vector, $q$, for the two copolymers in 466 $\mathrm{D}_{2} \mathrm{O}$ at different temperatures. Generally, concentration 467 fluctuations on a given length scale, i.e., a certain $q$ range, 468 lead to intensified scattering intensities. Both copolymer 469 solutions cause little scattering at $20{ }^{\circ} \mathrm{C}$, as expected for a 470 homogeneous polymer solution. 

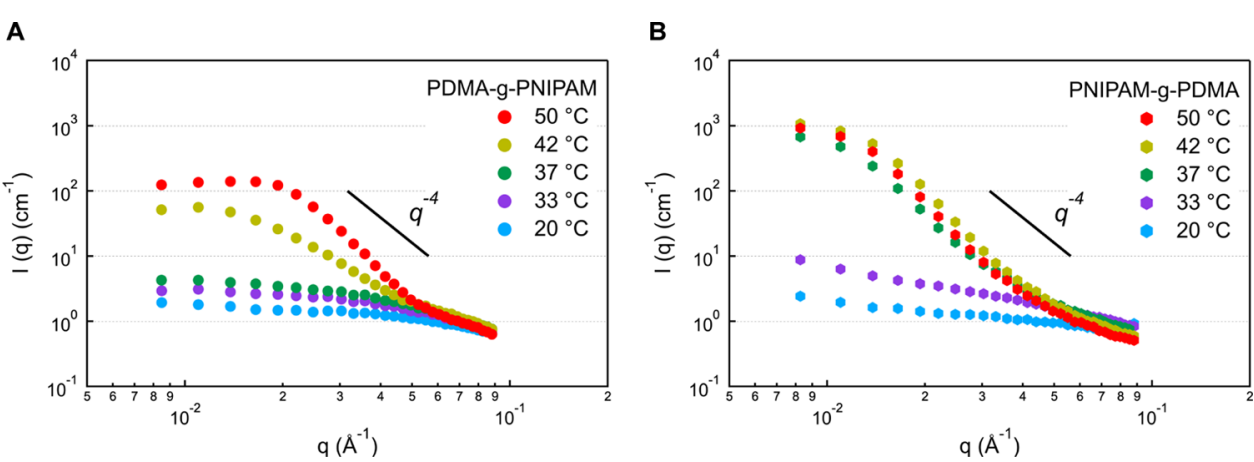

Figure 4. Evolution of the scattering intensity with temperature for $8 \mathrm{wt} \%$ (A) PDMA-g-PNIPAM and (B) PNIPAM- $g$-PDMA in $\mathrm{D}_{2} \mathrm{O}$. The solid lines mark $I \propto q^{-4}$.

472 In the case of PDMA- $g$-PNIPAM (Figure 4A), between 20 473 and $37{ }^{\circ} \mathrm{C}$, there is no significant variation of the scattering 474 intensity, $I(q)$, over the $q$ range studied. However, once the 475 transition temperature is crossed $\left(T>40{ }^{\circ} \mathrm{C}\right)$, the intensity 476 starts to grow steadily in the low $q$ range. Eventually, at $50{ }^{\circ} \mathrm{C}$, 477 the scattering intensity follows Porod's law in the asymptotic 478 regime (medium to high $q$ range), which predicts a quadratic 479 power-law dependence of the scattered intensity on the 480 scattering vector $\left(I \propto q^{-4}\right)$. This behavior, typical of phase 481 separation with sharp phase boundaries, here between 482 PNIPAM-rich- and PNIPAM-poor domains, has been 483 previously reported for similar physical ${ }^{30}$ and chemical ${ }^{29,31}$ 484 hydrogels as well as for other water-soluble polymers modified 485 with PNIPAM. ${ }^{45}$ At $50{ }^{\circ} \mathrm{C}$, a peak in intensity appears at a 486 given scattering vector, $q^{*}$, which corresponds to the 487 periodicity of the phase separation with a characteristic length, ${ }_{488} d_{c}=\frac{2 \pi}{q^{*}} \cong 450 \AA$. The peak in intensity is clearly visible on a 489 lin-lin plot, as shown in Figure S7.

490 On the other hand, the transition occurs much more 491 abruptly in the case of PNIPAM- $g$-PDMA (Figure 4B), with 492 the scattering intensity increasing significantly in the low $q$ 493 range as soon as the transition temperature is exceeded $(T>$ $49435^{\circ} \mathrm{C}$ ). In the meantime, agreement with Porod's law is 495 observed in the asymptotic regime, indicating that the system 496 features a two-phase morphology with sharp interfaces by 37 $497{ }^{\circ} \mathrm{C}$ with little change at higher temperatures. However, no clear 498 scattering peak can be distinguished for $q>8 \times 10^{-3} \AA^{-1}$. This 499 means that the phase separation takes place on a larger scale $500\left(d_{c} \geq 750 \AA\right)$ with the formation of larger PNIPAM-rich 501 domains (compared to the inverse topology).

502 The more gradual transition in the case of PDMA-g503 PNIPAM is in agreement with the broader transition observed 504 in DSC. The SANS results suggest the progressive formation of 505 isolated phase-separated domains (micellar structure) whose 506 boundaries become gradually sharper with temperature. This is 507 in line with the increased difficulty for smaller PNIPAM side 508 chains to enter aggregates as speculated from the DSC. On the 509 other hand, PNIPAM-g-PDMA shows a sharp transition with 510 the formation of PNIPAM-rich domains well in place by 37 $511{ }^{\circ} \mathrm{C}$. Due to the structure of the copolymer, which allows 512 entanglement formation between PNIPAM backbones in 513 semidilute solutions, we assume that the PNIPAM- $g$-PDMA 514 copolymer gives rise to a physical network with a percolating 515 PNIPAM phase above the transition temperature.

516 A qualitative analysis of the SANS data presented in Figure 517 S8 the SI was performed to estimate the specific surface (the 518 surface-to-volume ratio, $S_{\text {spe }}$ ) and the average composition of the PNIPAM-rich domains. For this purpose, the volume 519 fraction of PNIPAM $\left(\phi_{\text {NIPAM }}\right)$ in these domains was calculated 520 from Invariant using the contrast difference between the two 521 phases according to the following assumptions: (1) all of the 522 PNIPAM ends up into the hydrophobic domains with a 523 fraction of $\mathrm{D}_{2} \mathrm{O}\left(\phi_{\mathrm{D}_{2} \mathrm{O}}=1-\phi_{\text {PNIPAM }}\right)$ and (2) the remaining 524 volume is occupied by the swollen PDMA phase. These 525 assumptions are quite realistic as the findings of the analyses 526 are in good quantitative agreement with the previous 527 literature. $^{30,42}$ Table 3 summarizes the main data obtained 528 t3 from SANS experiments.

Table 3. Summary of SANS Calculations (see Figure S8 for Details)

\begin{tabular}{rrrcc} 
sample & $q^{*}\left(\AA^{-1}\right)$ & $d_{\mathrm{c}}(\AA)$ & $\begin{array}{c}\phi_{\mathrm{PNIPAM}} \\
(\text { vol \%) }\end{array}$ & $\begin{array}{c}S_{\text {spe }}=S_{2} / V_{2} \\
\left(\AA^{-1}\right)\end{array}$ \\
$\begin{array}{c}\text { PDMA-g- } \\
\text { PNIPAM }\end{array}$ & 0.014 & 450 & 72 & 0.036 \\
$\begin{array}{c}\text { PNIPAM-g- } \\
\text { PDMA }\end{array}$ & $\leq 0.008$ & $\geq 750$ & 76 & 0.014 \\
\hline
\end{tabular}

As reported in a previous paper, the volume fraction of 530 PNIPAM in the phase-separated domains at $50{ }^{\circ} \mathrm{C}$ is quite 531 high, between 70 and 80 vol \%. ${ }^{30}$ Nonetheless, it remains 532 below the glass transition temperature $\left(T_{\mathrm{g}}\right)$ which is reported 533 to be $132{ }^{\circ} \mathrm{C}$ for pure PNIPAM, $66{ }^{\circ} \mathrm{C}$ for $C_{\text {PNIPAM }}=88$ wt $\% 534$ $\left(C_{\mathrm{D}_{2} \mathrm{O}}=12 \mathrm{wt} \%\right)$ and $24{ }^{\circ} \mathrm{C}$ for $\mathrm{C}_{\mathrm{PNIPAM}}=76 \mathrm{wt} \%\left(\mathrm{C}_{\mathrm{D}_{2} \mathrm{O}}=24{ }_{535}\right.$ wt \%), respectively. ${ }^{25}$ This concentration, below but not far 536 from the glassy state, is in good agreement with the phase 537 diagram reported by Berghmans and co-workers and the low 538 dynamics of PNIPAM sequences involved in the aggregates as 539 already highlighted by NMR and viscoelastic experiments. ${ }^{42} 540$ The specific surfaces of the PNIPAM-rich domains were 541 calculated to be 0.036 and $0.014 \AA^{-1}$ in PDMA-g-PNIPAM 542 and PNIPAM-g-PDMA hydrogels, respectively. Given the 543 much larger specific area in the case of aggregates formed with 544 short PNIPAM side chains, which would correspond to a 545 radius $R=83 \AA$ assuming spherical objects $\left(S_{2} / V_{2}=3 / R\right)$, and 546 little difference in the water contents of the hydrophobic 547 domains between the two topologies, we ascribe the lower 548 transition enthalpy to a lower level of hydrogen bonds 549 disruption between NIPAM units and water molecules at the 550 periphery of these microdomains. Another consequence of this 551 distinction is evident in the level of transparency of the two 552 hydrogels at $50{ }^{\circ} \mathrm{C}$ (see Figure 1). The PDMA-g-PNIPAM 553 hydrogel is only slightly turbid, but the inverse topology 554 becomes completely opaque upon heating. This observation 555 
556 goes very well with the measured scattering intensities and the 557 corresponding sizes of the domains (see Figure 4 and Table 3 ). 558 3.3. Linear Rheology (Small Deformations). In this 559 part, we study the thermal transition of the inverse topologies 560 by means of linear rheology. Sufficiently small deformations in 561 this regime ensure no disruption of the gel microstructure or 562 chain conformation in solution, thus providing information 563 about the materials at rest. This assumption was confirmed via 564 stress amplitude sweeps as shown in Figure S3 in the SI. 565 3.3.1. Sol-Gel Transition. Figure 5 shows the temperature 566 dependence of the storage modulus, $G^{\prime}$, and the loss modulus,

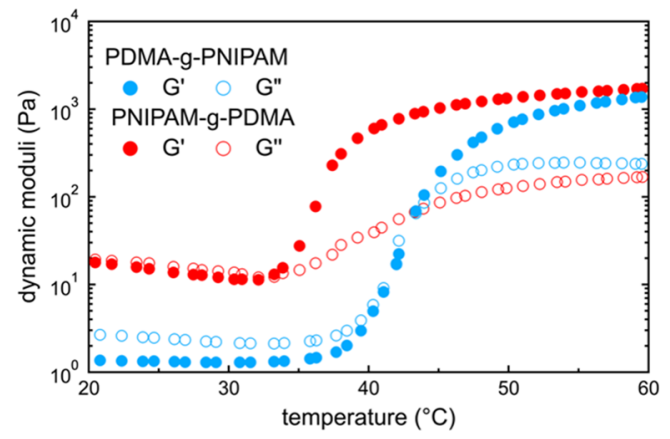

Figure 5. Thermal evolution of the storage $\left(G^{\prime}\right)$ and loss $\left(G^{\prime \prime}\right)$ moduli of 8 wt \% PDMA- $g$-PNIPAM and PNIPAM- $g$-PDMA at a heating rate of $2{ }^{\circ} \mathrm{C} \cdot \mathrm{min}^{-1}$.

$567 G^{\prime \prime}$, for 8 wt $\%$ copolymer solutions/hydrogels. The heating 568 rate was $2{ }^{\circ} \mathrm{C} \cdot \mathrm{min}^{-1}$. In the case of PDMA- $g$-PNIPAM, the 569 low-temperature behavior is characteristic of a viscous fluid $570\left(G^{\prime \prime}>G^{\prime}\right)$ and the moduli follow an Arrhenius dependence 571 with an activation energy of $26 \mathrm{~kJ} \cdot \mathrm{mol}^{-1}$, typical of a 572 nonassociating polymer aqueous solution in this range of 573 concentrations. ${ }^{46}$ The activation energy was calculated from the slope of $\ln \left(\eta^{*}\right)$ versus $1 / T$, where $T$ is the temperature in 574 kelvin and $\eta^{*}$ is the complex viscosity $\left|\eta^{*}\right|=\frac{1}{\omega} \times \sqrt{G^{\prime \prime}+G^{\prime 2}},{ }_{575}$ where $\omega$ is the angular frequency. In good agreement with the 576 DSC and SANS data, the moduli start to rise at $38{ }^{\circ} \mathrm{C} .577$ However, the gelation marked by the cross over of the dynamic 578 moduli $\left(G^{\prime}=G^{\prime \prime}\right)$ does not occur until $43{ }^{\circ} \mathrm{C} .{ }^{47}$ The moduli 579 then keep on rising until reaching a pseudoplateau by 50-55 580 ${ }^{\circ} \mathrm{C}$.

The inverse topology, i.e., PNIPAM- $g$-PDMA, has a similar 582 low-temperature $\left(<32{ }^{\circ} \mathrm{C}\right)$ behavior with an activation energy 583 of $27 \mathrm{~kJ} \cdot \mathrm{mol}^{-1}$. While $G^{\prime \prime}$ remains above $G^{\prime}$, this solution is 584 more viscoelastic $\left(G^{\prime \prime} \approx G^{\prime}\right)$ than the other with a complex 585 viscosity about 10 times higher. As expected from DSC and 586 SANS, the rise in the moduli occurs at $\sim 33{ }^{\circ} \mathrm{C}$. This is 587 followed, almost immediately, by the formation of a 588 percolating network leading to gelation. The transition is 589 also sharper, and a pseudoplateau is well in place by $45-45^{\circ} \mathrm{C} .590$

In both cases, the growth of the moduli above the transition 591 temperature accounts for the strengthening of hydrophobic 592 interactions at higher temperatures along with the disruption 593 of additional hydrogen bonds with water molecules. In other 594 words, by increasing the temperature above $T_{\text {onset }}$ the phase 595 separation process of the binary system PNIPAM/water leads 596 to an increasing number of PNIPAM chains that self-associate 597 into increasingly concentrated domains (corresponding to the 598 higher number of physical cross-links and longer lifetime of the 599 associations). A pseudoplateau is then reached when almost all 600 of the PNIPAM chains are segregated.

601

Since the copolymers are both hydrophilic below their 602 transition temperatures, the difference in their low-temperature 603 viscosities is due to the higher molecular weight of PNIPAM-g- 604 PDMA and its higher level of entanglement. However, far 605 above the transition, both hydrogels are soft viscoelastic solids 606
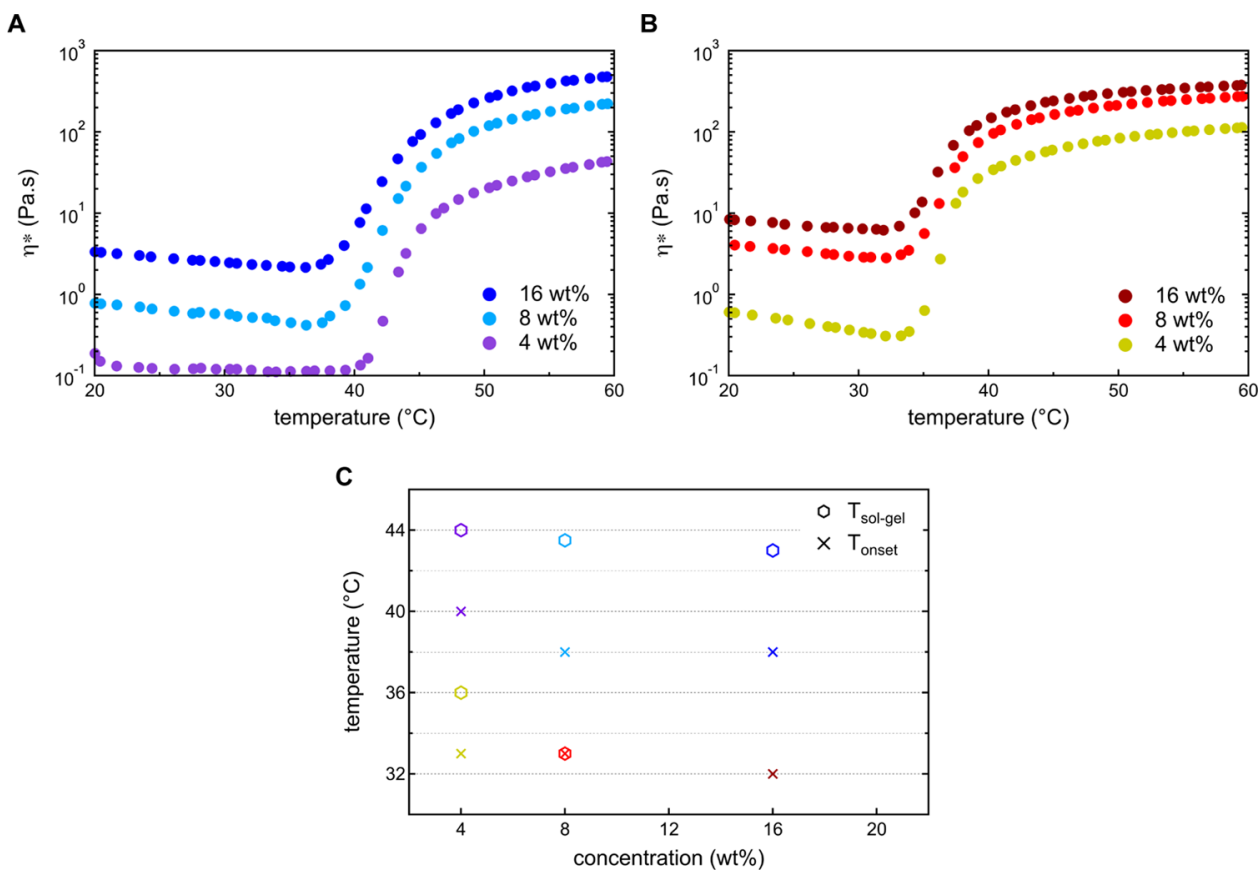

Figure 6. Temperature dependence of complex viscosity, $\eta^{*}$, for (A) PDMA- $g$-PNIPAM and (B) PNIPAM- $g$-PDMA solutions/hydrogels at the concentrations indicated in the legends and $(\mathrm{C})$ corresponding onset $\left(T_{\text {onset }}\right)$ and sol-gel $\left(T_{\text {sol-gel }}\right)$ temperatures. Note that no $T_{\text {sol-gel }}$ is reported in the case of $16 \mathrm{wt} \%$ PNIPAM-g-PDMA as it is already in the gel state at room temperature. The color code has been consistently applied. 

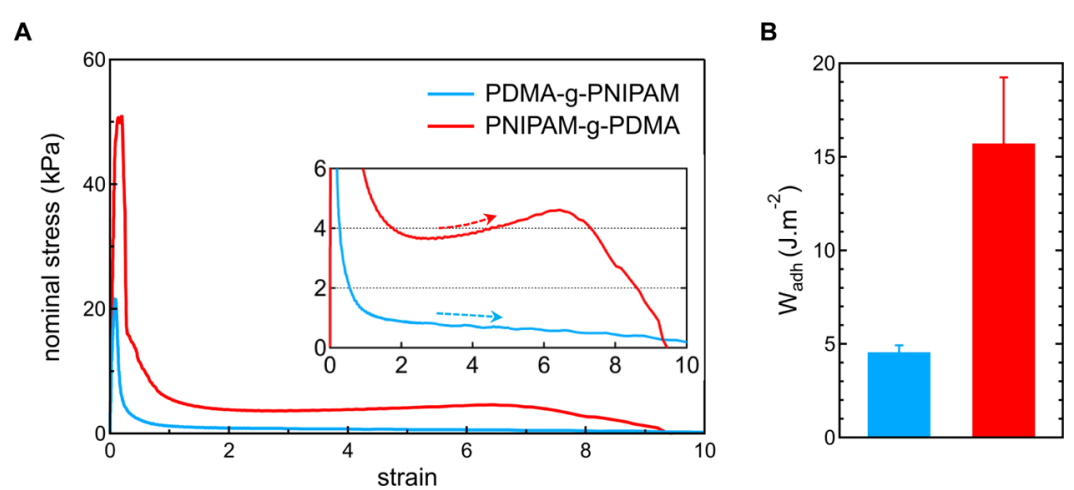

Figure 7. (A) Nominal stress-strain curves from probe tack experiments on $400 \mu \mathrm{m}$ thick layers of the hydrogels $\left(50{ }^{\circ} \mathrm{C}\right.$, after $\left.10 \mathrm{~min}\right)$ at $\dot{\varepsilon}=0.25$ $\mathrm{s}^{-1}$. The plateau in stress is magnified in the inset. (B) Corresponding adhesion energies $\left(W_{\text {adh }}\right)$ with standard deviation reported as error bars.

607 with comparable mechanical properties (the storage moduli in 608 the range of $1-2 \mathrm{kPa}$ ) in the linear regime.

609 At low deformations, it therefore appears that the 610 viscoelastic properties of the "composite" hydrogels formed 611 at high temperatures are strongly coupled with the properties 612 of the PNIPAM-rich domains forming the "hard" phase, while 613 the topology of the copolymers and the initial level of 614 entanglements seem to have a smaller impact under these 615 conditions. This finding is in agreement with previous studies 616 based on grafted copolymer solutions or grafted chemical gels. 617 Indeed, similar elastic moduli have been obtained for opposite 618 topologies having the same PNIPAM composition when 619 studied at the same copolymer concentration. ${ }^{29-31}$ Whatever 620 the biphasic morphology formed at high temperatures, there 621 would be a strong coupling of the viscoelastic properties of the 622 gels with the mechanical properties of the PNIPAM-rich phase. 623 It should be noted that these sol-to-gel transitions are 624 completely reversible and that the initial moduli (in the sol 625 state) are recovered when the hydrogels are cooled down at 626 the same rate, albeit with a small hysteresis of $1-2{ }^{\circ} \mathrm{C} .{ }^{30}$ This 627 in turn means that the solutions/hydrogels are reasonably close 628 to the equilibrium state throughout the experiment. The 629 viscoelastic response of the solutions (at $20^{\circ} \mathrm{C}$ ) and the 630 hydrogels (at $50{ }^{\circ} \mathrm{C}$ ) can be found in Figure S9.

631 3.3.2. Role of Polymer Concentration in Linear Viscoe632 lastic Properties. For simplicity, the thermal association 633 behavior of the samples prepared at different polymer 634 concentrations is compared based on a single viscoelastic 635 parameter, i.e., the complex viscosity, as shown in Figure 6A,B 636 for PDMA- $g$-PNIPAM and PNIPAM- $g$-PDMA solutions/ 637 hydrogels, respectively. The corresponding data in terms of 638 dynamic moduli can be found in Figure S10 in the SI. Figure $6396 \mathrm{C}$ plots the onset of the transition ( $T_{\text {onset }}$ defined as the 640 temperature corresponding to a $10 \%$ deviation from the 641 Arrhenius dependence) and the gelation point $\left(T_{\text {sol-gel }}\right.$ as 642 defined previously).

643 Taking into account that below $T_{\text {onset }}$ the complex viscosities 644 are more than 50 times higher than the viscosity of the solvent $645\left(10^{-3} \mathrm{~Pa} \cdot \mathrm{s}\right.$ at $\left.20^{\circ} \mathrm{C}\right),{ }^{48}$ we can assume that the solutions are 646 already in the entangled regime at these concentrations. Then, 647 the evolution of $\eta^{*}$ generally follows the same trends as those 648 described for each topology at 8 wt \%. At low temperatures, 649 the polymers form viscous solutions in water with a similar 650 Arrhenius dependence $\left(E_{\eta} \cong 26 \mathrm{~kJ} \cdot \mathrm{mol}^{-1}\right)$. Above the thermal 651 transition $\left(T_{\text {onset }}\right)$, the phase separation of PNIPAM residues 652 leads to the initial rise in complex viscosity, which grows by $2-$ 6533 decades with temperature.
A close inspection of the difference between onset and sol- 654 gel temperatures, given in Figure 6C, offers additional insights 655 into the associating behavior. All PDMA-g-PNIPAM solutions 656 feature a weak concentration dependence of these character- 657 istic temperatures with a wide transition zone $\left(T_{\text {sol-gel }}-T_{\text {onset }} 658\right.$ $=4-5{ }^{\circ} \mathrm{C}$ ), in agreement with DSC experiments. This means 659 that, within this temperature range, PNIPAM domains begin to 660 form across the sample, but the level of association should be 661 high enough for PDMA chains to bridge PNIPAM-rich 662 domains. On the other hand, while PNIPAM-g-PDMA 663 solutions also display a weak concentration dependence of 664 the onset temperature, the viscoelastic transition is much 665 sharper and the sol-gel temperatures are strongly correlated to 666 the initial viscoelastic behavior of the copolymer solution. 667 Indeed, the higher molar mass of PNIPAM-g-PDMA is 668 responsible for its higher level of entanglement and elasticity 669 compared to PDMA-g-PNIPAM. For instance, the solution at 670 16 wt \% already exhibits $G^{\prime} \cong G^{\prime \prime}$ at room temperature, below 671 the transition temperature of PNIPAM.

If we only consider the association process, all of the results 673 obtained at rest or at low deformation by DSC, SANS, and 674 linear rheology highlight the significance of the molar mass of 675 PNIPAM, independently of the copolymer topology, on the 676 onset temperature as well as on the sharpness of the transition. 677 This conclusion is supported by a comparison with the work of 678 Guo et al. ${ }^{30}$ who studied these inverse topologies with a similar 679 composition (50:50) in the linear rheological regime. The 680 transition temperature with their PDMA-g-PNIPAM is $3{ }^{\circ} \mathrm{C} 681$ lower than that of ours regardless of the concentration. This is 682 because the PNIPAM side chains used in their work are 683 roughly 3 times larger than ours, supporting the significance of 684 the molecular weight of the PNIPAM in the gelation behavior 685 of these responsive copolymers. With the inverse topology 686 (PNIPAM-g-PDMA), however, we find the same transition 687 temperatures because the thermoresponsive backbones in both 688 cases are already quite large $\left(M_{\mathrm{n}}>250 \mathrm{~kg} \cdot \mathrm{mol}^{-1}\right)$.

In summary, the two systems exhibit similar behavior in the 690 linear regime with a slight difference for PDMA-g-PNIPAM 691 which self-associates at a higher temperature and over a wider 692 temperature range. At 16 wt \%, PNIPAM- $g$-PDMA is too 693 viscous to manipulate with a micropipette, which could 694 possibly undermine its contact with substrates. Moreover, the 695 gain in moduli when the concentration is increased from 8 to 696 16 wt $\%$ is not substantial. At the lower limit, i.e., 4 wt \%, the 697 solutions are easily injectable, but the gels formed are basically 698 too soft and the gaps between their moduli, too wide. For these 699 
700 reasons, the main focus of the nonlinear mechanical studies 701 will be on 8 wt \% samples.

702 3.4. Nonlinear Mechanical Properties (Large Defor703 mations). All nonlinear experiments were performed at $50{ }^{\circ} \mathrm{C}$ 704 to avoid complications arising from evaporation. However, to 705 show that the results would be similar at $60{ }^{\circ} \mathrm{C}$, an example of 706 a probe tack experiment at $60{ }^{\circ} \mathrm{C}$ will be provided later.

707 3.4.1. Probe Tack. 3.4.1.1. Role of Copolymer Topology. 708 Figure $7 \mathrm{~A}$ compares the nominal stress-strain curves from 709 probe tack experiments on $400 \mu \mathrm{m}$ thick layers of the 710 hydrogels at a constant debonding rate of $100 \mu \mathrm{m} \cdot \mathrm{s}^{-1}$ 711 (corresponding to a nominal strain rate of $0.25 \mathrm{~s}^{-1}$ ) after 10 $712 \mathrm{~min}$ at $50{ }^{\circ} \mathrm{C}$. The inset magnifies the stress plateau region. 713 The corresponding adhesion energies are given in Figure $7 \mathrm{~B}$. 714 The two curves are generically similar in small strains $(\varepsilon<1)$ : 715 the nominal stress builds up to a peak as the layer is 716 homogeneously deformed much the same way as in a confined 717 tensile test. The sharp peak in stress is higher for the PNIPAM$718 \mathrm{~g}$-PDMA hydrogel, which was anticipated from the dynamic 719 moduli.

720 Both hydrogels are then stretched to large deformations 721 through the formation of fibrillar structures, as seen in the 722 lateral images in Figure 8A,B taken during debonding.
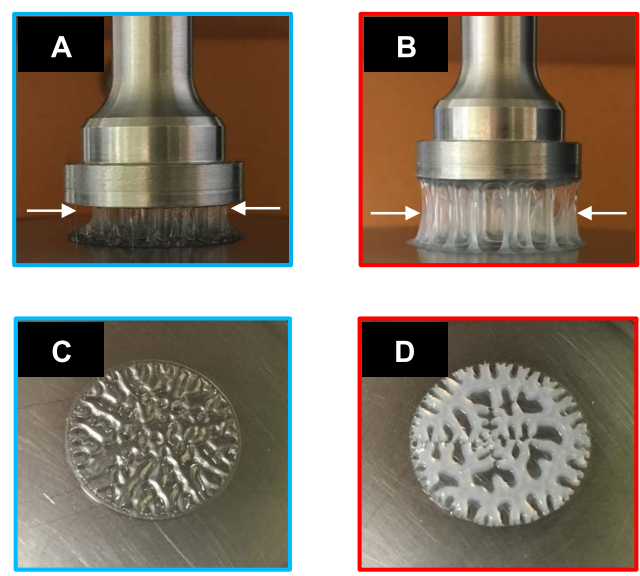

Figure 8. Fibrillation during debonding of (A) PDMA- $g$-PNIPAM and (B) PNIPAM- $g$-PDMA. In both cases, the failure was cohesive: in (A), failure started close to the interface with the probe, moving from the periphery toward the center, whereas in (B), failure occurred within the filaments after ultimate deformation of the layer. $(C, D)$ Images of the layers immediately after debonding.

723 Extensive fibrillation is in fact a prerequisite to obtaining 724 such strains (as large as 1000\%) during debonding of confined, 725 incompressible layers. Nonetheless, the shape of the stress726 strain curves reveals inherent differences in nonlinear 727 mechanical properties. Upon stretching, filaments of the 728 PDMA-g-PNIPAM hydrogel continually soften as shown by 729 the steady decrease in nominal stress (marked by the blue 730 arrow in the inset of Figure 7A). With the inverse topology, 731 however, the stress initially goes through a plateau during fibril 732 formation and then rises again during stretching to large 733 deformations (marked by the red arrow in the inset of Figure $7347 \mathrm{~A}$ ). This suggests the possibility of strain hardening in the 735 filaments.

736 We postulate that the different nonlinear behaviors of the 737 two hydrogels, i.e., strain softening versus strain hardening, are 738 due to their phase-separated morphologies determined by their 739 topologies. It may be argued that the slightly lower storage modulus of PDMA- $g$-PNIPAM at $50{ }^{\circ} \mathrm{C}$ is responsible for this 740 distinction. However, a comparison of probe tack properties of 741 PDMA-g-PNIPAM at $60{ }^{\circ} \mathrm{C}$ with that of PNIPAM- $g$-PDMA at 742 $50{ }^{\circ} \mathrm{C}$ in Figure $\mathrm{S} 11$ in the SI invalidates this scenario.

743

This topology-dependent difference in nonlinear behavior 744 results in a measured work of adhesion, which is more than 3745 times larger for the PNIPAM backbone as shown in Figure 7B. 746 The stress-strain behavior of this hydrogel, i.e., PNIPAM-g- 747 PDMA (Figure 7A, red curve), remarkably resembles that of a 748 classic hydrophobic PSA, albeit at lower stress levels. We note 749 that such $\mathrm{W}_{\mathrm{adh}}$ values are, at least, 1 order of magnitude lower 750 compared to soft commercial hydrophobic adhesives, since 751 these adhesive hydrogels are very soft. ${ }^{49}$ For comparison, in a 752 commercial PSA, the initial cavitation peak is on the order of 753 0.1-1 MPa with the plateau stress remaining on the order of 754 $50 \%$ of the initial peak. ${ }^{15,50}$

Without the possibility of direct visual observation from the 756 bottom plate in our experiments, it is not straightforward to 757 unambiguously determine the debonding mechanisms such as 758 the possibility of cavitation. Yet, the shapes of the layers after 759 complete detachment, shown in Figure $8 \mathrm{C}, \mathrm{D}$, provide some 760 insight. The marked drop in stress following the initial peak 761 (Figure 7A) coincides with the occurrence of fingering 762 instabilities in the periphery of the hydrogels (Figure 8C,D). 763 In general, these instabilities appearing as fingerlike protrusions 764 of a low-viscosity fluid (e.g., air) are pushed into a second 765 medium of higher viscosity (e.g., viscous oils, soft liquidlike 766 adhesives) in confinement. ${ }^{51-53}$ In tension, the outer contact 767 line destabilizes as air (low-viscosity medium) penetrates the 768 fluidlike layer (high-viscosity medium) that is sucked inward. 769 This phenomenon has been widely reported in the literature 770 for confined layers of various fluids (Newtonian, yield stress, 771 viscoelastic, etc.), ${ }^{51,53,54}$ but also for soft elastic solids where 772 fingering instabilities maximize the compliance of the layer. ${ }^{55,56} 773$

Shull and Creton ${ }^{50}$ have proposed that bulk fingering occurs 774 in confined layers of soft, elastic solids when $r / h_{0}>2$ (where $r 775$ and $h_{0}$ are the radius and thickness of the layer, respectively) 776 and relatively large ratios $(>10)$ of atmospheric pressure to 777 Young's modulus $\left(P_{0} / E\right)$. In practice, the second criterion 778 satisfies the suppression of internal cavities by the external 779 pressure. We can thus attribute the formation of the fingers to 780 the highly confined geometry of the layer $\left(r / h_{0}=25\right)$ and the 781 very soft nature of the gels $\left(G^{\prime} \sim 1 \mathrm{kPa}\right)$. These instabilities 782 appear around the peak in nominal stress at the adhesive-air 783 interface. As the probe is pulled up, their growth into the bulk 784 provides the initial deformation of the adhesive layer, leading 785 to a large drop in stress. Subsequently, the walls of the fingers 786 are stretched into fibrils, allowing the hydrogels to go through 787 extensive deformation (Figure 8A,B). ${ }^{53,57}$

788

In both cases, the fingering instabilities form two sets of 789 regular patterns entering the layer from the edge, one with 790 bigger instabilities at a larger wavelength intertwined by smaller 791 fingers. In the case of the PDMA-g-PNIPAM hydrogel, the 792 fingers seem to grow radially preferentially closer to the 793 interface with the probe based on the wrinkled appearance of 794 the top of the layer. This means that air penetrates radially 795 close to the probe without causing much deformation in the 796 bulk. As a consequence, the filaments come off following the 797 propagation path of the fingers: starting at the periphery 798 toward the center (Figure 8A).

799

With the PNIPAM-g-PDMA hydrogel, however, the fingers 800 grow only a limited distance toward the center with the biggest 801 ones traveling $\sim 10-20 \%$ of the layer's radius $(r)$. Instead, the 802 
C
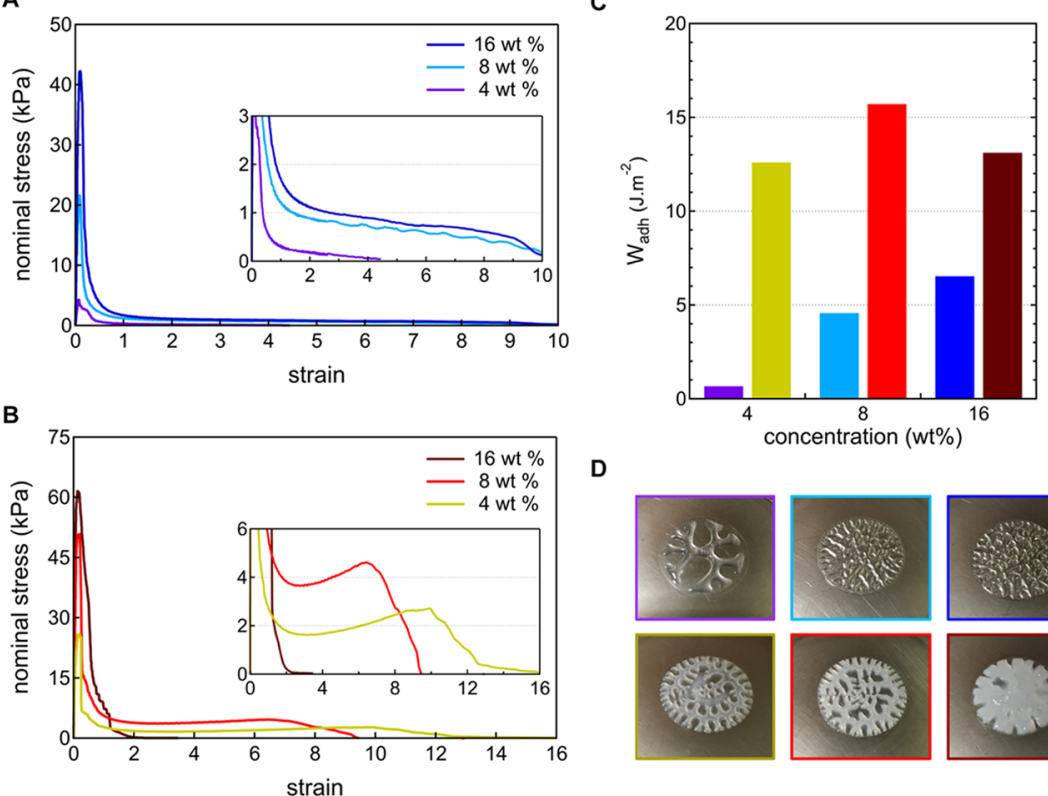

D

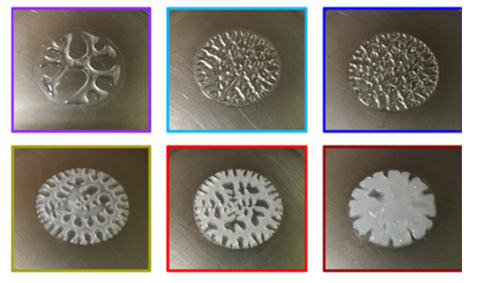

Figure 9. Nominal stress-strain curves from probe tack experiments $\left(50{ }^{\circ} \mathrm{C}\right.$, after $10 \mathrm{~min}, h_{0}=400 \mu \mathrm{m}$, and $\left.\dot{\varepsilon}=0.25 \mathrm{~s}^{-1}\right)$ on hydrogels containing 4, 8, and 16 wt \% (A) PDMA-g-PNIPAM and (B) PNIPAM-g-PDMA. The insets magnify the plateau regions. (C) Corresponding adhesion energies $\left(W_{\text {adh }}\right)$. (D) Images of the debonded layers immediately after detachment. The color code has been consistently applied.

803 central part of the adhesive appears poked with other 804 instabilities that resemble open cavities. One may argue that 805 level of the peak stress $(\sim 50 \mathrm{kPa})$ falls below that of 806 atmospheric pressure, which would preclude the possibility of 807 bulk cavitation. However, it should be noted that this value is 808 an average over the entire area of the layer. The distribution of 809 the tensile stress in a highly confined, incompressible layer is 810 parabolic due to lateral stresses arising from confinement, with 811 the maximum (equal to twice the average value) occurring at 812 the center. ${ }^{33,34,55}$ It is thus likely that the stress level at the 813 center of the probe is on the order of or slightly higher than 814 atmospheric pressure $(0.1 \mathrm{MPa})$, allowing trapped air bubbles 815 to grow and form cavities in the bulk of the adhesive. This 816 suggests that this soft adhesive might be just at the limit of bulk 817 cavitation at this strain rate. In all likelihood, the pressure in 818 these cavities is equilibrated very quickly $(\varepsilon<2)$, leading to an 819 open cell structure as the filaments grow out of the popped 820 cavity walls and the peripheral fingers (Figure $8 \mathrm{~B}$ ).

821 These arguments are backed by probe tack tests on layers 822 with different thicknesses or levels of confinement, as shown in 823 Figure S12. The confinement level at half the thickness $\left(r / h_{0}=\right.$ $82450)$ results in a peak stress exceeding $0.1 \mathrm{MPa}$ with cavities 825 clearly visible over most of the layer's surface after the test. We 826 note that the strain rate is not the same in these experiments. 827 By increasing the level of confinement, we validated the 828 occurrence of cavitation in the PNIPAM- $g$-PDMA hydrogel.

829 The interpretations so far are based on probe tack 830 experiments on hydrogels at $8 \mathrm{wt} \%$ polymer under a given 831 set of experimental conditions. The following sections 832 investigate the effects of polymer concentration and testing 833 geometry to see whether the distinction in strain-hardening 834 behavior can be characterized by a more conventional 835 nonlinear rheological test.

836 3.4.1.2. Role of Polymer Concentration in Probe Tack.

837 Figure 9A,B plots the nominal stress-strain curves from probe 838 tack experiments on gels containing 4, 8, and 16 wt \% PDMA$839 \mathrm{~g}$-PNIPAM and PNIPAM- $g$-PDMA, respectively. The exper- imental conditions are identical to those in the previous part 840 for $8 \mathrm{wt} \%$ hydrogels (see Figure 7 ) and so is the level of 841 confinement $\left(r / h_{0}=25\right)$. The adhesion energies as a function 842 of polymer concentration are compared in Figure 9C. To 843 complement our analysis, postfailure images of all of the layers 844 are displayed in Figure 9D. With either topology, the peak 845 stress consistently increases with polymer concentrations, as 846 expected from the parallel trend in the storage modulus (see 847 temperature sweeps in Figure S10 in the SI). In other words, 848 fingering instabilities appear at lower stress levels in less 849 concentrated hydrogels.

850

The 4 wt \% PDMA-g-PNIPAM hydrogel, although a stable 851 gel according to linear rheology $\left(G^{\prime}>G^{\prime \prime}\right.$, Figure 7 in the SI), 852 is quite soft $\left(G^{\prime} \sim 100 \mathrm{~Pa}, \eta^{*} \sim 20 \mathrm{~Pa} \cdot \mathrm{s}\right)$ and behaves much 853 like a viscous fluid in probe tack, featuring a small peak in 854 nominal stress followed by quick softening and detachment by 855 a strain of 4 . This is consistent with the pattern of the 856 debonded layer (Figure 9D), suggesting the presence of the so- 857 called Saffman-Taylor instabilities typically observed in 858 confined layers of liquids. $^{51,52,58}$ At 8 and 16 wt \%, though, 859 the fingers lead up to fibrillation resulting in extensive 860 deformation of the bulk of the layer up to strains of 10 . We 861 emphasize that strain softening is observed in the fibrillation 862 plateau at all of the concentrations studied. Stress levels simply 863 increase with concentration, suggesting an increase in viscosity. 864 This is manifested in the corresponding adhesion energies in 865 Figure 9C.

866

The mechanical behavior of the 4 wt \% PNIPAM- $g$-PDMA 867 hydrogel is generically similar to its $8 \mathrm{wt} \%$ counterpart. The 868 fact that it is more stretchable is due to the lower degree of 869 physical cross-linking at this concentration. This difference is 870 qualitatively comparable to lowering the (chemical) cross- 871 linking degree in soft hydrophobic PSAs. ${ }^{59-61}$ Note that 872 already at a polymer concentration of $4 \mathrm{wt} \%$, the work of 873 adhesion exceeds $12 \mathrm{~J} \cdot \mathrm{m}^{-2}$, while that of the inverse topology 874 (with the liquidlike behavior) is less than $1 \mathrm{~J} \cdot \mathrm{m}^{-2}$. Finally, 875 fibrillation is not observed at 16 wt $\%$ (with $\varepsilon_{\max } \approx 2$ ). This is 876 
877 typical of early failure through adhesive detachment from the 878 probe and consistent with limited elastic instabilities in the 879 periphery and none in the bulk (Figure 9D). Here, fibril 880 formation is hindered by the increased elastic modulus of the 881 gel. ${ }^{56}$ We also note that this sample is already at the gel point 882 at room temperature (see Figure S10 in the SI), which can 883 adversely affect contact formation with the probe.

884 In conclusion, our results highlight a nontrivial tradeoff 885 between injectability and adhesiveness as a function of polymer 886 concentration. Beyond the given concentration, (1) the 887 solutions become either noninjectable and/or too viscous to 888 be able to make effective contact with adherents in short 889 contact times and (2) the hydrogels become too stiff to allow 890 extensive bulk deformation before detachment from the probe. 891 Both of these effects, seen with the 16 wt \% PNIPAM- $g$-PDMA 892 hydrogel, compromise the final adhesion energy (see Figure 893 9C).

894 3.4.2. Stress Growth. Figure 10A plots the stress-strain 895 curves for $8 \mathrm{wt} \%$ hydrogels sheared in the rheometer at a

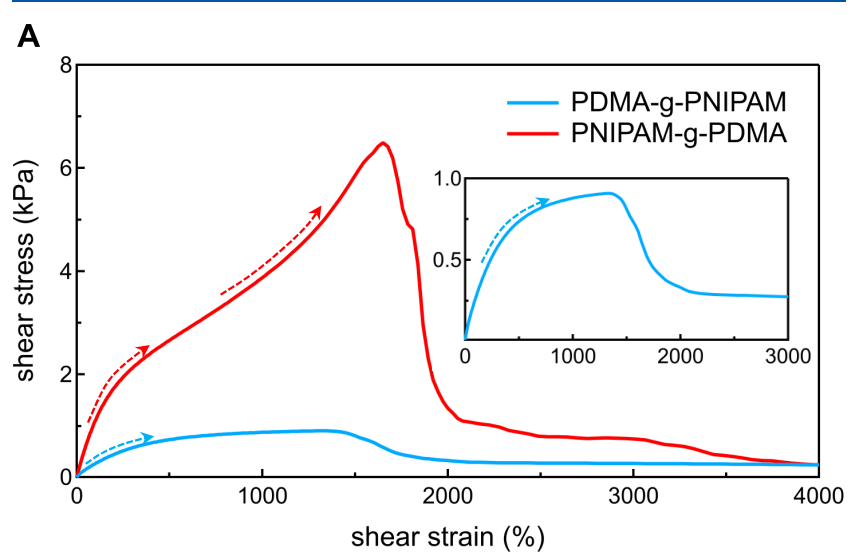

B

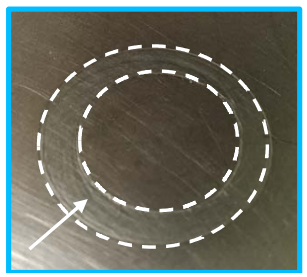

C

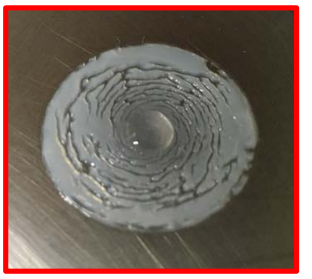

Figure 10. (A) Shear stress versus shear strain from stress growth experiments at a constant rate of $0.25 \mathrm{~s}^{-1}$ on $8 \mathrm{wt} \%$ PDMA- $g$ PNIPAM and PNIPAM- $g$-PDMA hydrogels at $50{ }^{\circ} \mathrm{C}$. The inset magnifies the curve for PDMA-g-PNIPAM. (B, C) Postfailure images of the hydrogels. The sample in (B) is in the space between the dashed circles, as the central part was removed with the cone. constant shear rate of $0.25 \mathrm{~s}^{-1}$ up to and beyond failure. The 896 postfailure images from the rheometer plate for PDMA-g- 897 PNIPAM and PNIPAM-g-PDMA are shown in Figure 10B,C, 898 respectively. As expected, the values of the modulus obtained 899 from the initial slope (evaluated below 20\% strain) of these 900 curves, 0.37 and $1.40 \mathrm{kPa}$, respectively, correspond well with 901 the storage moduli obtained from linear rheology experiments. 902

Nevertheless, what is observed in Figure 10A goes beyond a 903 mere difference in the softness of the two hydrogels. It is only 904 the PDMA-g-PNIPAM hydrogel that softens continuously up 905 to failure $(\sim 1400 \%)$. The image taken immediately after the 906 experiment (Figure 10B) reveals no visible evidence of shear 907 bands or rupture, suggesting that the gel started to flow on a 908 microscopic scale upon softening. On the other hand, when 909 PNIPAM forms the backbone, the hydrogel displays, after the 910 initial softening, a hardening regime evident in the sharp 911 upturn of the stress beyond $\sim 700 \%$ of strain. The gel clearly 912 fractures in the bulk along with the formation of shear bands 913 (Figure 10C) above $\sim 1700 \%$. Similar shear bands were 914 microscopically observed in model protein gels by Keshavarz 915 et al. ${ }^{17}$

916

As shown in Figure S13 in the SI, the two hydrogels 917 maintain their characteristic nonlinear behaviors at shear rates 918 between 0.025 and $0.25 \mathrm{~s}^{-1}$. The hardening observed in one 919 hydrogel and not in the other stresses the importance of the 920 copolymer topology, as previously seen with probe tack 921 experiments (see Figure 8). In other words, the difference in 922 nonlinear behavior of the inverse topologies is an intrinsic 923 material effect and is not due to the specific loading geometry. 924 In the following, we use this information to discuss the possible 925 morphology of the hydrophobic aggregates acting as cross- 926 linking points in the gels.

927

3.5. Nanostructure-Property Relationships. Our DSC 928 and SANS data suggest differences in morphology of the 929 phase-separated domains between the two hydrogels. In 930 particular, PNIPAM-g-PDMA has an abrupt transition with 931 an enthalpy comparable to a homopolymer PNIPAM, while its 932 inverse topology having the same PNIPAM content as side 933 chains is characterized by a more gradual transition with less 934 than half the enthalpy (see Figure 3). Yet, the two hydrogels 935 reach the same storage moduli far above their thermal 936 transition temperatures (see Figure 5), suggesting that the 937 linear viscoelastic properties of these gels are mainly 938 determined by the average composition (of the soft and hard 939 (PNIPAM-rich) phases) rather than by the morphology. 940

We then performed nonlinear mechanical experiments in 941 large deformations to help distinguish their nanostructures. 942 Both probe tack and stress growth experiments (see Figures 8943 and 11) revealed a hardening behavior unique to the $944 \mathrm{f} 11$
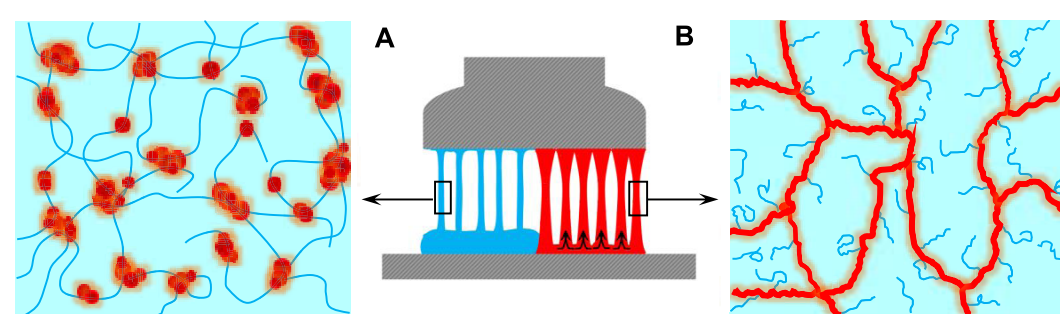

Figure 11. Schematic nanostructures proposed for (A) PDMA-g-PNIPAM and (B) PNIPAM- $g$-PDMA hydrogels at $50{ }^{\circ} \mathrm{C}$. The cartoon in the middle represents the fibrillation stage of the respective probe tack experiments. Strain hardening with the hydrophobic nanoscaffold leads to stable fibrils and deformation of the entire layer. Strain softening with discrete hydrophobic clusters leads to unstable fibrils only deforming the top-most part of the layer. 
945 PNIPAM-g-PDMA hydrogel. The inverse topology consis946 tently showed softening at all of the concentrations (see Figure 947 9), temperatures (see Figure S11), and shear rates (see Figure 948 S13 in the SI) studied. It may be argued that the softening of 949 the PDMA-g-PNIPAM hydrogel is due to the lower molecular 950 weight (Table 1) and thus the lower degree of entanglements 951 of this copolymer. However, this is not the case since the $8 \mathrm{wt}$ $952 \%$ solution of PDMA-g-PNIPAM, which is roughly as 953 entangled as the $4 \mathrm{wt} \%$ solution of PNIPAM-g-PDMA 954 (given that they differ in size by a factor of 2 , see Table 1 ), 955 shows no hardening (Figure 9A,B). In fact, the work of 956 adhesion of 16 wt \% PDMA-g-PNIPAM is only half that of the 957 inverse topology at $4 \mathrm{wt} \%$ (Figure 9C). Therefore, the 958 properties of these hydrogels are controlled by the association 959 of PNIPAM moieties into hydrophobic domains rather than by 960 the molecular weight of the copolymer. Therefore, what 961 matters is the length of PNIPAM in each copolymer that 962 determines the final morphology of the associations.

963 To explain the topology-specific nonlinear behavior of the 964 two hydrogels, we propose the nanostructures shown in Figure 965 11. In the case of PDMA- $g$-PNIPAM, short thermoresponsive 966 grafts may self-associate into discrete, concentrated clusters 967 (containing $\sim 28$ vol \% water content, see Table 3 ). PDMA 968 chains between PNIPAM associations would then act as 969 elastically active bridges. The more gradual transition and 970 delayed gelation (by $4-5{ }^{\circ} \mathrm{C}$ ) in this case may be due to a 971 nucleation and growth mechanism of these associations with 972 the early ones acting as the nuclei which grow in size by the 973 incorporation of more NIPAM side chains over time. This is 974 consistent with the observations made on analogous chemical 975 hydrogels. ${ }^{29}$

976 In contrast, PNIPAM-g-PDMA contains very long, already 977 entangled PNIPAM backbones whose collapse leads to 978 immediate gelation as they become hydrophobic. As 979 mentioned earlier, the only plausible mechanism for this to 980 occur is the formation of a dense ( 24 vol \% water), 981 hydrophobic nanophase of PNIPAM percolating the swollen 982 PDMA matrix in a bicontinuous morphology. ${ }^{32}$ We note that 983 the hydrophobic domains only occupy $5-6 \%$ of the total 984 volume of the hydrogel based on SANS data (Figure 4 and 985 Table 3).

986 In both cases, the mechanical properties and stability of the 987 hydrogels may be due to the very slow dynamics of PNIPAM 988 in the aggregates. Note that these hydrogels do not have a well989 defined relaxation time measurable within the time scale of our 990 experiments (see Figure S9). In large strains, load transfer 991 occurs via elastically active PDMA chains bridging associations 992 of short chains in the case of the PDMA- $g$-PNIPAM hydrogel 993 and via very long, heavily entangled PNIPAM backbones 994 (within the hydrophobic nanoscaffold) in the case of the 995 PNIPAM- $g$-PDMA hydrogel. ${ }^{62}$ On a single-molecule level, the 996 energy barrier against pulling a longer hydrophobic chain out 997 of an association domain is far larger for long chains than for 998 shorter ones.

999 The occurrence of strain hardening is consistent with a 1000 percolating nanoscaffold of strong hydrophobic associations 1001 capable of effective load transfer in different directions, as was 1002 seen in both elongation and shear for PNIPAM-g-PDMA (see 1003 Figures 7 and 11). Under similar conditions, isolated domains 1004 simply deform, e.g., from spherical to ellipsoidal shapes, until 1005 stress is relaxed via chain pullout, resulting in strain softening. 1006 Therefore, in the first case, fibrillation deforms the entire bulk 1007 of the layer, while in the second case, it simply deforms the top-most part of the layer near the interface with the probe 1008 (Figure 8).

Such nanophase morphologies leading to similar linear 1010 properties but distinctive nonlinear behaviors have been 1011 previously proposed for chemical gels based on the same 1012 chemistry as well as PSAs based on block copolymers. ${ }^{29,63}$ For 1013 the chemical gels, the fracture energies of the PNIPAM-g- 1014 PDMA hydrogels are an order of magnitude higher than their 1015 inverse topologies, ${ }^{27,31}$ which was ascribed to the bifurcation of 1016 the propagating crack as its tip reached oriented percolating 1017 PNIPAM-rich domains. ${ }^{27}$

The bicontinuous nanostructure proposed in Figure 11 is 1019 also consistent with single-molecule simulations on similar 1020 graft copolymers. ${ }^{64}$ For a very long backbone and sparsely 1021 spaced grafts, the simulation shows the evolution of spherical 1022 micelles into cylindrical ones as the quality of the solvent for 1023 the backbone was reduced (this is similar to the effect of 1024 temperature on PNIPAM in our work). Given that our 1025 solutions are in the semidilute regime (well above the $c^{*}$ ) and 1026 arguably above the entanglement concentration $\left(c_{\mathrm{e}}\right)$ (see 1027 Figure 6), we can reasonably believe that entangled PNIPAM 1028 backbones self-associate and collapse into percolating 1029 PNIPAM-rich domains stabilized by hydrophilic coronae of 1030 swollen PDMA side chains, as schematized in Figure 11B.

1031

\section{CONCLUSIONS}

We investigated the linear and nonlinear mechanical properties 1032 of two injectable hydrogels, PDMA- $g$-PNIPAM and PNIPAM- 1033 g-PDMA, with inverse topologies. At $50{ }^{\circ} \mathrm{C}$ (above the LCST 1034 of PNIPAM), we found that, while linear properties were 1035 indistinguishable, adhesive properties in probe tack experi- 1036 ments were markedly different. The PNIPAM-g-PDMA 1037 hydrogel, with long, highly entangled PNIPAM backbones, 1038 showed significant hardening behavior in large strains. The 1039 hydrogel based on the inverse topology bearing much shorter 1040 PNIPAM side chains only showed softening in large strains. 1041 This important distinction was observed at all concentrations 1042 where the solutions were injectable $\left(G^{\prime \prime}>G^{\prime}\right)$, at different 1043 levels of mechanical confinement (layer thickness), as well as 1044 in shear deformation (stress growth experiments), and was 1045 thus characteristic of the specific copolymer topology.

1046

We linked this topology-specific distinction in the nonlinear 1047 mechanical properties of the hydrogels to their nanostructures. 1048 The hardening behavior was associated with a continuous 1049 nanostructure consisting of strong hydrophobic PNIPAM-rich 1050 domains. The softening of the inverse topology was due to the 1051 easier pullout of PNIPAM grafts from separate hydrophobic 1052 associations bridged via PDMA backbones. The insensitivity of 1053 linear rheology to such nanostructural differences suggests that 1054 the viscoelastic properties of these hydrogels are mainly 1055 controlled by the average composition (of the soft and hard 1056 phases) rather than by the morphology.

1057

Finally, while the best adhesive properties were obtained 1058 when long and entangled LCST chains collapse to form a 1059 continuous hard phase, the performance optimization relies on 1060 a subtle balance involving not only the topology but also the 1061 monomer composition as well as the chemistry of the 1062 responsive polymer. The latter factor strongly impacts the 1063 binding energy within the hard phase and thereby the bulk 1064 mechanical properties, as highlighted by our work. These key 1065 ideas pave the way for future developments of injectable 1066 hydrogels with generic adhesive properties in aqueous 1067 environments. 
(4) Weiden, J.; Voerman, D.; Dölen, Y.; Das, R. K.; Van Duffelen, 1125

A.; Hammink, R.; Eggermont, L. J.; Rowan, A. E.; Tel, J.; Figdor, C. 1126

G. Injectable Biomimetic Hydrogels as Tools for Efficient T Cell 1127

Expansion and Delivery. Front. Immunol. 2018, 9, No. 2798.

(5) Dimatteo, R.; Darling, N. J.; Segura, T. In Situ Forming 1129 Injectable Hydrogels for Drug Delivery and Wound Repair. Adv. Drug 1130 Delivery Rev. 2018, 127, 167-184.

1131

(6) Van Tomme, S. R.; Storm, G.; Hennink, W. E. In Situ Gelling 1132 Hydrogels for Pharmaceutical and Biomedical Applications. Int. J. 1133 Pharm. 2008, 355, 1-18.

1134

(7) Ghobril, C.; Grinstaff, M. W. The Chemistry and Engineering of 1135 Polymeric Hydrogel Adhesives for Wound Closure: A Tutorial. Chem. 1136 Soc. Rev. 2015, 44, 1820-1835.

1137

(8) Yang, J.; Bai, R.; Chen, B.; Suo, Z. Hydrogel Adhesion: A 1138 Supramolecular Synergy of Chemistry, Topology, and Mechanics. 1139 Adv. Funct. Mater. 2019, No. 1901693.

1140

(9) Scognamiglio, F.; Travan, A.; Rustighi, I.; Tarchi, P.; Palmisano, 1141 S.; Marsich, E.; Borgogna, M.; Donati, I.; De Manzini, N.; Paoletti, S. 1142 Adhesive and Sealant Interfaces for General Surgery Applications. J. 1143 Biomed. Mater. Res., Part B 2016, 104, 626-639.

(10) Duarte, A. P.; Coelho, J. F.; Bordado, J. C.; Cidade, M. T.; Gil, 1145 M. H. Surgical Adhesives: Systematic Review of the Main Types and 1146 Development Forecast. Prog. Polym. Sci. 2012, 37, 1031-1050. 1147

(11) Stuart, M. A. C.; Huck, W. T. S.; Genzer, J.; Müller, M.; Ober, 1148 C.; Stamm, M.; Sukhorukov, G. B.; Szleifer, I.; Tsukruk, V. V.; Urban, 1149 M.; Winnik, F.; Zauscher, S.; Luzinov, I.; Minko, S. Emerging 1150 Applications of Stimuli-Responsive Polymer Materials. Nat. Mater. 1151 2010, 9, 101-113.

(12) Gil, E. S.; Hudson, S. M. Stimuli-Reponsive Polymers and 1153 Their Bioconjugates. Prog. Polym. Sci. 2004, 29, 1173-1222. 1154

(13) Roy, D.; Cambre, J. N.; Sumerlin, B. S. Future Perspectives and 1155 Recent Advances in Stimuli-Responsive Materials. Prog. Polym. Sci. 1156 2010, 35, 278-301.

(14) Kamperman, M.; Synytska, A. Switchable Adhesion by 1158 Chemical Functionality and Topography. J. Mater. Chem. 2012, 22, 1159 19390. 1160

(15) Creton, C.; Ciccotti, M. Fracture and Adhesion of Soft 1161 Materials: A Review. Rep. Prog. Phys. 2016, 79, No. 046601.

(16) Webber, R. E.; Creton, C.; Brown, H. R.; Gong, J. P. Large 1163 Strain Hysteresis and Mullins Effect of Tough Double-Network 1164 Hydrogels. Macromolecules 2007, 40, 2919-2927.

1165

(17) Keshavarz, B.; Divoux, T.; Manneville, S.; McKinley, G. H. 1166 Nonlinear Viscoelasticity and Generalized Failure Criterion for 1167 Polymer Gels. ACS Macro Lett. 2017, 6, 663-667.

(18) Bai, R.; Yang, Q.; Tang, J.; Morelle, X. P.; Vlassak, J.; Suo, Z. 1169 Fatigue Fracture of Tough Hydrogels. Extreme Mech. Lett. 2017, 15, 1170 91-96.

1171

(19) Ruel-Gariépy, E.; Leroux, J. C. In Situ-Forming Hydrogels - 1172 Review of Temperature-Sensitive Systems. Eur. J. Pharm. Biopharm. 1173 2004, 58, 409-426.

1174

(20) Teotia, A. K.; Sami, H.; Kumar, A. Thermo-Responsive 1175 Polymers: Structure and Design of Smart Materials. Switchable and 1176 Responsive Surfaces and Materials for Biomedical Applications; Elsevier 1177 Ltd., 2015; pp 3-43.

1178

(21) Durand, A.; Hervé, M.; Hourdet, D. Thermogelation in 1179 Aqueous Polymer Solutions. In Stimuli-Responsive Water Soluble and 1180 Amphiphilic Polymers; McCormick, C. L., Eds.; ACS Publications, 1181 2000; pp 181-207.

(22) Aseyev, V.; Tenhu, H.; Winnik, F. M. Non-ionic Thermores- 1183 ponsive Polymers in Water. In Self Organized Nanostructures of 1184 Amphiphilic Block Copolymers II; Müller, A.; Borisov, O., Eds.; 1185 Advances in Polymer Science; 2011; Vol. 242, pp 29-89. 1186

(23) Schild, H. G. Poly(N-Isopropylacrylamide): Experiment, 1187 Theory and Application. Prog. Polym. Sci. 1992, 17, 163-249. 1188

(24) Otake, K.; Inomata, H.; Konno, M.; Saito, S. Thermal Analysis 1189 of the Volume Phase Transition with N-Isopropylacrylamide Gels. 1190 Macromolecules 1990, 23, 283-289.

(25) Liu, P.; Song, L.; Li, N.; Lin, J.; Huang, D. Time Dependence 1192 of Phase Separation Enthalpy Recovery Behavior in Aqueous Poly(N- 1193 
1194 Isopropylacrylamide) Solution. J. Therm. Anal. Calorim. 2017, 130, $1195843-850$.

1196 (26) Shibayama, M.; Morimoto, M.; Nomura, S. Phase Separation 1197 Induced Mechanical Transition of Poly(N-Isopropylacrylamide)/ 1198 Water Isochore Gels. Macromolecules 1994, 27, 5060-5066.

1199 (27) Guo, H.; Sanson, N.; Hourdet, D.; Marcellan, A. Thermores1200 ponsive Toughening with Crack Bifurcation in Phase-Separated 1201 Hydrogels under Isochoric Conditions. Adv. Mater. 2016, 28, 7043. 1202 (28) Chassenieux, C.; Nicolai, T.; Benyahia, L. Rheology of 1203 Associative Polymer Solutions. Curr. Opin. Colloid Interface Sci. 1204 2011, 16, 18-26.

1205 (29) Guo, H.; Mussault, C.; Brûlet, A.; Marcellan, A.; Hourdet, D.; 1206 Sanson, N. Thermoresponsive Toughening in LCST-Type Hydrogels 1207 with Opposite Topology: From Structure to Fracture Properties. 1208 Macromolecules 2016, 49, 4295-4306.

1209 (30) Guo, H.; Brûlet, A.; Rajamohanan, P. R.; Marcellan, A.; Sanson, 1210 N.; Hourdet, D. Influence of Topology of LCST-Based Graft 1211 Copolymers on Responsive Assembling in Aqueous Media. Polymer 1212 2015, 60, 164-175.

1213 (31) Guo, H.; Sanson, N.; Marcellan, A.; Hourdet, D. Thermores1214 ponsive Toughening in LCST-Type Hydrogels: Comparison between 1215 Semi-Interpenetrated and Grafted Networks. Macromolecules 2016, 1216 49, 9568-9577.

1217 (32) Vahdati, M.; Ducouret, G.; Creton, C.; Hourdet, D. Thermally 1218 Triggered Injectable Underwater Adhesives. Macromol. Rapid 1219 Commun. 2020, 41, No. 1900653.

1220 (33) Lakrout, H.; Sergot, P.; Creton, C. Direct Observation of 1221 Cavitation and Fibrillation in a Probe Tack Experiment on Model 1222 Acrylic Pressure-Sensitive-Adhesives. J. Adhes. 1999, 69, 307-359.

1223 (34) Creton, C.; Lakrout, H. Micromechanics of Flat-Probe 1224 Adhesion Tests of Soft Viscoelastic Polymer Films. J. Polym. Sci., 1225 Part B: Polym. Phys. 2000, 38, 965-979.

1226 (35) Zheng, X.; Tong, Z.; Xie, X.; Zeng, F. Phase Separation in 1227 Poly(N-Isopropyl Acrylamide)/Water Solutions I. Cloud Point 1228 Curves and Microgelation. Polym. J. 1998, 30, 284-288.

1229 (36) Schild, H. G.; Tirrell, D. A. Microcalorimetric Detection of 1230 Lower Critical Solution Temperatures in Aqueous Polymer Solutions. 1231 J. Phys. Chem. A. 1990, 94, 4352-4356.

1232 (37) Petit, L.; Karakasyan, C.; Pantoustier, N.; Hourdet, D. 1233 Synthesis of Graft Polyacrylamide with Responsive Self-Assembling 1234 Properties in Aqueous Media. Polymer 2007, 48, 7098-7112.

1235 (38) Petit, L.; Bouteiller, L.; Brûlet, A.; Lafuma, F.; Hourdet, D. 1236 Responsive Hybrid Self-Assemblies in Aqueous Media. Langmuir 1237 2007, 23, 147-158.

1238 (39) Song, L.; Lin, J.; Liu, P.; Li, J.; Jiang, S.; Huang, D. Quantitative 1239 Determination of the Spring Entropy Effect and Its Indication of the 1240 Conformational Change of Polymer Coils with Varying Concen1241 tration in Aqueous Poly(N-Isopropylamide) Solutions. RSC Adv. 1242 2019, 9, 5540-5549.

1243 (40) Rao, K. K.; Rao, K. K.; Ha, C.-S. Stimuli Responsive Poly(Vinyl 1244 Caprolactam) Gels for Biomedical Applications. Gels 2016, 2, No. 6. 1245 (41) Durand, A.; Hourdet, D. Synthesis and Thermoassociative 1246 Properties in Aqueous Solution of Graft Copolymers Containing 1247 Poly(N-Isopropylacrylamide) Side Chains. Polymer 1999, 40, 494112484951.

1249 (42) Afroze, F.; Nies, E.; Berghmans, H. Phase Transitions in the 1250 System Poly(N-Isopropylacrylamide)/Water and Swelling Behaviour 1251 of the Corresponding Networks. J. Mol. Struct. 2000, 554, 55-68.

1252 (43) Bokias, G.; Vasilevskaya, V. V.; Iliopoulos, I.; Hourdet, D.; 1253 Khokhlov, A. R. Influence of Migrating Ionic Groups on the Solubility 1254 of Polyelectrolytes: Phase Behavior of Ionic Poly(N-Isopropylacryla1255 mide) Copolymers in Water. Macromolecules 2000, 33, 9757-9763. 1256 (44) Shibayama, M.; Tanaka, T.; Han, C. C. Small Angle Neutron 1257 Scattering Study on Poly(N-Isopropyl Acrylamide) Gels near Their 1258 Volume-Phase Transition Temperature. J. Chem. Phys. 1992, 97, 1259 6829-6841.

1260 (45) Hourdet, D.; L'alloret, F.; Durand, A.; Lafuma, F.; Audebert, 1261 R.; Cotton, J. P. Small-Angle Neutron Scattering Study of Microphase
Separation in Thermoassociative Copolymers. Macromolecules 1998, 1262

9297, 5323-5335. 1263

(46) Guo, H.; De Magalhaes Goncalves, M.; Ducouret, G.; Hourdet, 1264 D. Cold and Hot Gelling of Alginate-Graft-PNIPAM: A Schizo- 1265 phrenic Behavior Induced by Potassium Salts. Biomacromolecules 1266 2018, 19, 576-587.

1267

(47) Winter, H. H.; Mours, M. Rheology of Polymers Near Liquid- 1268 Solid Transitions. Adv. Polym. Sci. 1997, 134, 165-234. 1269

(48) Dobrynin, A. V.; Colby, R. H.; Rubinstein, M. Scaling Theory 1270 of Polyelectrolyte Solutions. Macromolecules 1995, 28, 1859-1871. 1271

(49) Villey, R.; Creton, C.; Cortet, P. P.; Dalbe, M. J.; Jet, T.; 1272 Saintyves, B.; Santucci, S.; Vanel, L.; Yarusso, D. J.; Ciccotti, M. Rate- 1273 Dependent Elastic Hysteresis during the Peeling of Pressure Sensitive 1274 Adhesives. Soft Matter 2015, 11, 3480-3491. 1275

(50) Shull, K. R.; Creton, C. Deformation Behavior of Thin, 1276 Compliant Layers under Tensile Loading Conditions. J. Polym. Sci., 1277 Part B: Polym. Phys. 2004, 42, 4023-4043. 1278

(51) Amar, M. B.; Bonn, D. Fingering Instabilities in Adhesive 1279 Failure. Phys. D: Nonlinear Phenom. 2005, 209, 1-16. 1280

(52) Biggins, J. S.; Wei, Z.; Mahadevan, L. Fluid-Driven Fingering 1281 Instability of a Confined Elastic Meniscus. EPL 2015, 110, No. 34001.1282

(53) Nase, J.; Derks, D.; Lindner, A. Dynamic Evolution of 1283 Fingering Patterns in a Lifted Hele-Shaw Cell. Phys. Fluids 2011, 23, 1284 No. 123101.

1285

(54) Derks, D.; Lindner, A.; Creton, C.; Bonn, D. Cohesive Failure 1286 of Thin Layers of Soft Model Adhesives under Tension. J. Appl. Phys. 1287 2003, 93, 1557-1566.

(55) Webber, R. E.; Shull, K. R.; Roos, A.; Creton, C. Effects of 1289 Geometric Confinement on the Adhesive Debonding of Soft Elastic 1290 Solids. Phys. Rev. E 2003, 68, No. 021805.

1291

(56) Crosby, A. J.; Shull, K. R.; Lakrout, H.; Creton, C. Deformation 1292 and Failure Modes of Adhesively Bonded Elastic Layers. J. Appl. Phys. 1293 2000, 88, 2956-2966.

1294

(57) Nase, J.; Creton, C.; Ramos, O.; Sonnenberg, L.; Yamaguchi, 1295 T.; Lindner, A. Measurement of the Receding Contact Angle at the 1296 Interface between a Viscoelastic Material and a Rigid Surface. Soft 1297 Matter 2010, 6, 2685-2691.

1298

(58) Saffman, P. G.; Taylor, G. I. The Penetration of a Fluid into a 1299 Porous Medium or Hele-Shaw Cell Containing a More Viscous 1300 Liquid. Proc. R. Soc. London, Ser. A 1958, 245, 312-329. 1301

(59) Zosel, A. The Effect of Fibrilation on the Tack of Pressure 1302 Sensitive Adhesives. Int. J. Adhes. Adhes. 1998, 18, 265-271. 1303

(60) Zosel, A. Effect of Cross-Linking on Tack and Peel Strength of 1304 Polymers. J. Adhes. 1991, 34, 201-209.

1305

(61) Deplace, F.; Carelli, C.; Mariot, S.; Retsos, H.; Chateauminois, 1306 A.; Ouzineb, K.; Creton, C. Fine Tuning the Adhesive Properties of a 1307 Soft Nanostructured Adhesive with Rheological Measurements. J. 1308 Adhes. 2009, 85, 18-54.

1309

(62) Gunari, N.; Balazs, A. C.; Walker, G. C. Force-Induced 1310 Globule-Coil Transition in Single Polystyrene Chains in Water. J. Am. 1311 Chem. Soc. 2007, 129, 10046-10047.

1312

(63) Creton, C.; Hu, G.; Deplace, F.; Morgret, L.; Shull, K. R. Large- 1313 Strain Mechanical Behavior of Model Block Copolymer Adhesives. 1314 Macromolecules 2009, 42, 7605-7615.

1315

(64) Košovan, P.; Kuldová, J.; Limpouchová, Z.; Procházka, K.; 1316 Zhulina, E. B.; Borisov, O. V. Amphiphilic Graft Copolymers in 1317 Selective Solvents: Molecular Dynamics Simulations and Scaling 1318 Theory. Macromolecules 2009, 42, 6748-6760. 